\title{
Syndioselective 3,4-Polymerization of 1-Phenyl-1,3-Butadiene by Rare-earth Metal Catalysts
}

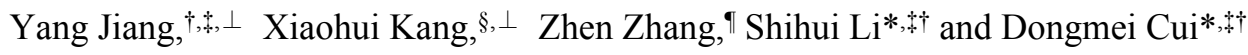 \\ $\dagger$ Ms. Y. Jiang, Dr. S. H. Li, Prof. D. M. Cui., State Key Laboratory of Polymer Physics and Chemistry, Changchun \\ Institute of Applied Chemistry, Chinese Academy of Sciences, Changchun 130022, China. \\ $\$$ Ms. Y. Jiang, School of Applied Chemistry and Engineering, University of Science and Technology of China. Hefei \\ 230026, China. \\ $\S$ Dr. X. H. Kang, College of Pharmacy, Dalian Medical University, Dalian 116044, China. \\ 『 Mr. Z. Zhang, Department of Materials Science and Engineering, Jilin University, Changchun 130022, China. \\ * E-mail: shihui-li@ciac.ac.cn \\ * E-mail: dmcui@ciac.ac.cn
}

\section{Contents}

Experimental Procedures .S3

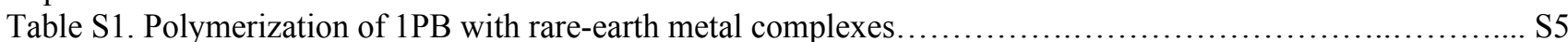

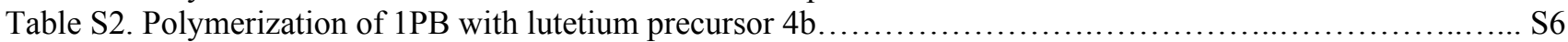

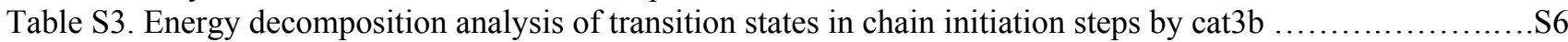

Table S4. Energy decomposition analysis of transition states in chain propagation processes ....................... 6

Table S5. Energy decomposition analysis of transition states in chain initiation steps by cat5 ...................S6

Table S6. Energy decomposition analysis of transition states in chain propagation processes by cat5 .............S7

Table S7. Energy decomposition analysis of transition states in chain propagation processes by cat7 and cat $8 \ldots . . . \mathrm{S} 7$

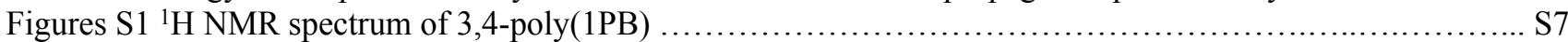

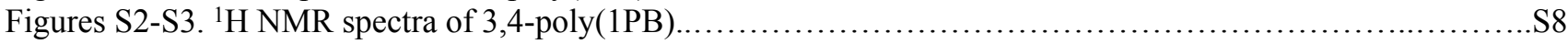

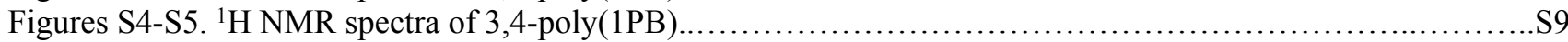

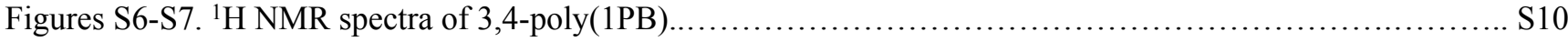

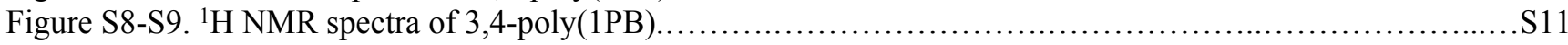

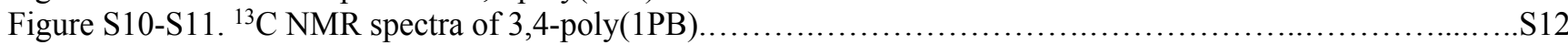

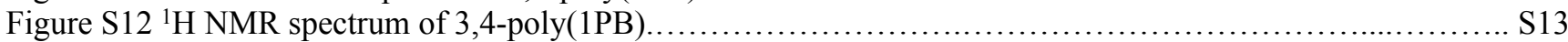

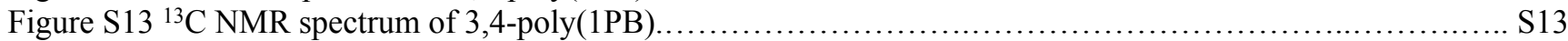

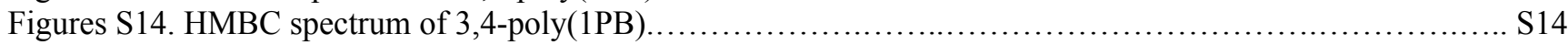

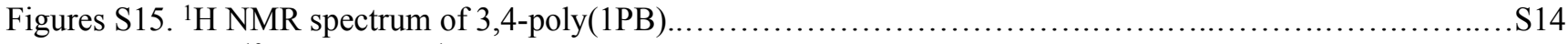

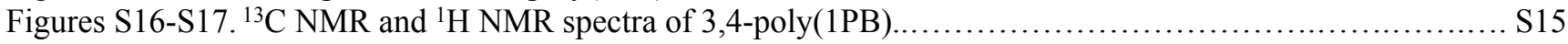

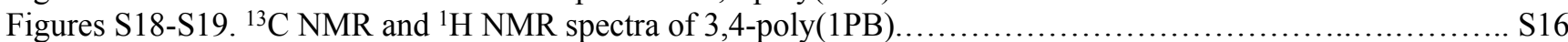

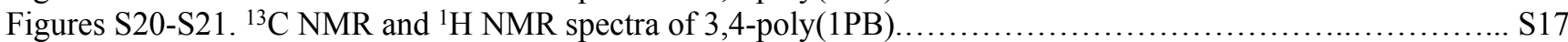

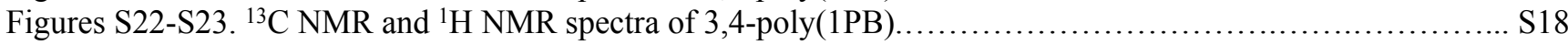

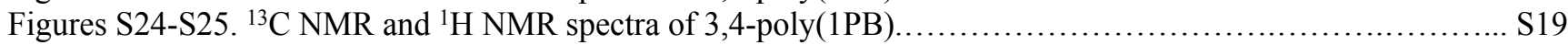

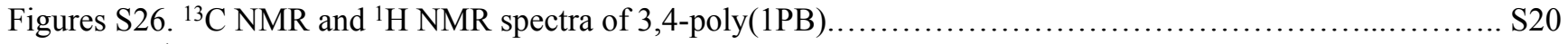

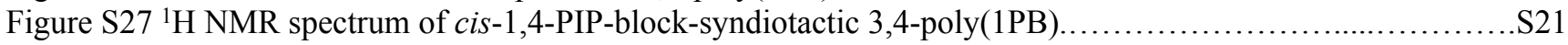

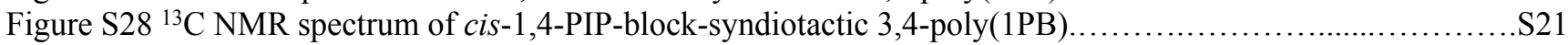

Figure S29 ${ }^{1} \mathrm{H}$ NMR spectra of syndiotactic 3,4-poly(1PB), hydrogenated syndiotactic 3,4-poly(1PB) and cyclized

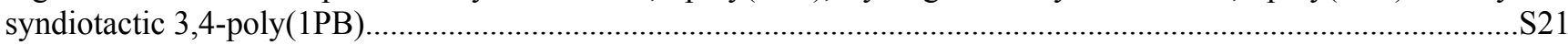

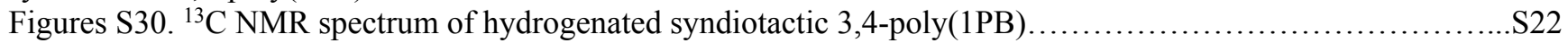

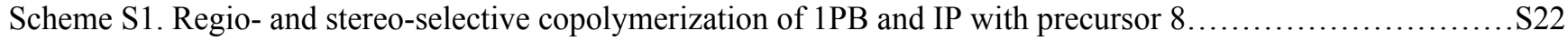

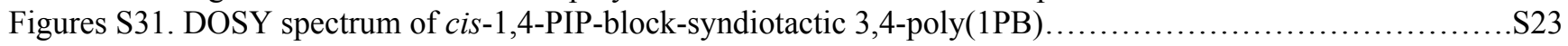

Figure S32. Diffusion coefficient from DOSY spectrum of of cis-1,4-PIP-block-syndiotactic 3,4-poly(1PB)........S23

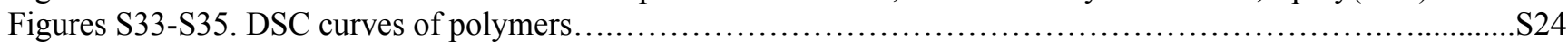


Figures S36. DSC curve of cis-1,4-PIP-block-syndiotactic 3,4-poly(1PB).

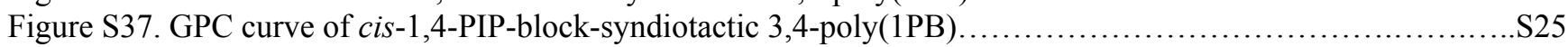

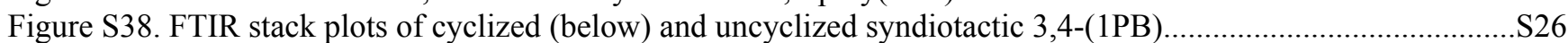

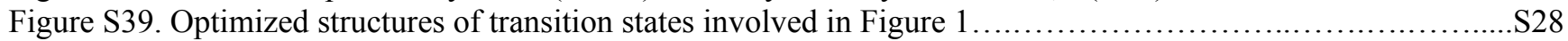

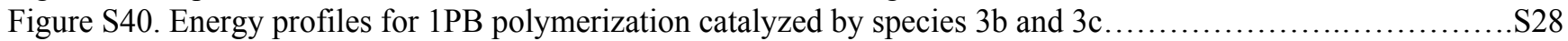

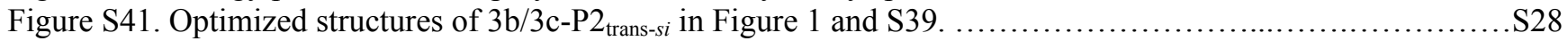

Figure S42. Energy profiles (A), key structures (B) and Optimized structures of transition states $(\mathbf{C})$ of the 1PB

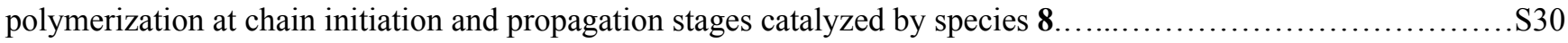

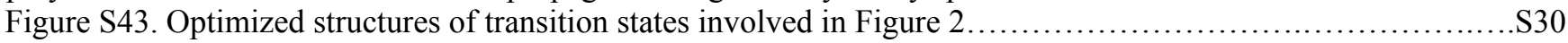




\section{Experimental Procedures}

General considerations. All manipulations were performed under a dry and oxygen-free nitrogen atmosphere using standard high-vacuum Schlenk techniques or in a glovebox. All solvents were purified via a SPS system. $\left[\mathrm{Ph}_{3} \mathrm{C}\right]\left[\mathrm{B}\left(\mathrm{C}_{6} \mathrm{~F}_{5}\right)_{4}\right], \mathrm{B}\left(\mathrm{C}_{6} \mathrm{~F}_{5}\right)_{3}$ are purchased from Energy Chemical. The monomers were dried over $\mathrm{CaH}_{2}$ under stirring for 12 hours and distilled before use. Complex 1-5 were prepared according to the reported methods. ${ }^{1}$

${ }^{1} \mathrm{H},{ }^{13} \mathrm{C}$, Dept ${ }^{135} \mathrm{NMR}$ spectra were recorded on a Bruker AV400 $\left(400 \mathrm{MHz}\right.$ for ${ }^{1} \mathrm{H} ; 100 \mathrm{MHz}$ for $\left.{ }^{13} \mathrm{C}\right)$ spectrometer. The molecular weight $\left(M_{\mathrm{n}}\right)$ was measured by TOSOH HLC-8220 GPC at $40{ }^{\circ} \mathrm{C}$ using THF as eluent (the flow rate is $0.35 \mathrm{~mL} / \mathrm{min})$ against polystyrene standards. The glass transition temperature $\left(T_{\mathrm{g}}\right)$ of the polymer was measured through differential scanning calorimetry (DSC) analysis, which was carried out on a METTLER TOPEM DSC instrument under nitrogen atmosphere. Any thermal history difference in the polymers was eliminated by first heating the specimen to above $200{ }^{\circ} \mathrm{C}$, cooling at $50{ }^{\circ} \mathrm{C} / \mathrm{min}$ to room temperature, and then recording the second DSC scan from $-50 / 25$ to $150 / 200 / 350{ }^{\circ} \mathrm{C}$ at $10^{\circ} \mathrm{C} / \mathrm{min}$.

Synthesis of 1-phenyl-1,3-butadiene (1PB). Under nitrogen, n-butyllithium ( $0.10 \mathrm{~mol}, 40 \mathrm{~mL}, 2.5 \mathrm{M}$ in hexane) was added dropwise to a suspension of methyltriphenylphosphonium bromide $(35.7 \mathrm{~g}, 0.10 \mathrm{~mol})$ in dry THF $(400 \mathrm{~mL})$ at $0{ }^{\circ} \mathrm{C}$ for $30 \mathrm{~min}$, followed by allowing to react at $0{ }^{\circ} \mathrm{C}$ for additional $2 \mathrm{~h}$. Then cinnamaldehyde $(11.9 \mathrm{~g}, 0.09 \mathrm{~mol})$ was added dropwise and the reaction mixture was allowed to react at room temperature for additional $6 \mathrm{~h}$. The reaction mixture was then neutralized with saturated $\mathrm{NH}_{4} \mathrm{Cl}$, extracted with petroleum ether, dried over $\mathrm{Na}_{2} \mathrm{SO}_{4}$, filtered, and concentrated in vacuo. Flash chromatography (petroleum) affords the pure product in a yield of $91 \%$. ${ }^{1} \mathrm{H}$ NMR (400 $\left.\mathrm{MHz}, \mathrm{CDCl}_{3}, 25^{\circ} \mathrm{C}\right): 5.17\left(\mathrm{~d}, 1 \mathrm{H}, \mathrm{CH}_{2}\right), 5.32\left(\mathrm{~d}, 1 \mathrm{H}, \mathrm{CH}_{2}\right), 6.45-6.58(\mathrm{~m}, 2 \mathrm{H}, \mathrm{CH}), 6.75-6.81(\mathrm{~m}, 1 \mathrm{H}, \mathrm{CH}), 7.22(\mathrm{t}, 1 \mathrm{H}$, $\left.p-\mathrm{C}_{6} \mathrm{H}_{5}\right), 7.31\left(\mathrm{t}, 2 \mathrm{H}, m-\mathrm{C}_{6} \mathrm{H}_{5}\right), 7.40\left(\mathrm{~d}, 2 \mathrm{H}, o-\mathrm{C}_{6} \mathrm{H}_{5}\right)$.

1PB Polymerization. A typical polymerization procedure (Table 1, run 4) was described as follow. Under nitrogen atmosphere, $1 \mathrm{~PB}(0.195 \mathrm{~g}, 1.5 \mathrm{mmol})$ was added into a $10 \mathrm{~mL}$ flask. Then, 10 equivalents of $\mathrm{Al}^{i} \mathrm{Bu}_{3}(0.20 \mathrm{~mL}, 0.5$ $\mathrm{mol} / \mathrm{L})$, a toluene solution $(1.0 \mathrm{~mL})$ of complex $3 \mathbf{a}(4.7 \mathrm{mg}, 10 \mu \mathrm{mol})$, and 1 equivalent of $\left[\mathrm{Ph}_{3} \mathrm{C}\right]\left[\mathrm{B}\left(\mathrm{C}_{6} \mathrm{~F}_{5}\right)_{4}\right](9.2$ $\mathrm{mg}, 10 \mu \mathrm{mol}$ ) were added to the flask at $25{ }^{\circ} \mathrm{C}$. After the reaction was stirred for $60 \mathrm{~min}$, methanol was injected to terminate the polymerization. The mixture was poured into a large quantity of methanol $(50 \mathrm{~mL})$. The obtained white polymer was filtered and then dried under vacuum at $40{ }^{\circ} \mathrm{C}$ to a constant weight.

Copolymerization of IP and 1PB. Under nitrogen atmosphere, 1PB (0.65 g, $5.0 \mathrm{mmol})$ and IP $(0.34,5.0 \mathrm{mmol})$ were added into a $10 \mathrm{~mL}$ flask. Then, 10 equivalents of $\mathrm{Al}^{i} \mathrm{Bu}_{3}(0.20 \mathrm{~mL}, 0.5 \mathrm{~mol} / \mathrm{L})$ in toluene, a toluene solution $(3.0 \mathrm{~mL})$ of complex $5(9.0 \mathrm{mg}, 10 \mu \mathrm{mol})$, and 1 equivalent of $\left[\mathrm{Ph}_{3} \mathrm{C}\right]\left[\mathrm{B}\left(\mathrm{C}_{6} \mathrm{~F}_{5}\right)_{4}\right](9.2 \mathrm{mg}, 10 \mu \mathrm{mol})$ were added to the flask at $25^{\circ} \mathrm{C}$. After the reaction was stirred for $150 \mathrm{~min}$, methanol was injected to terminate the polymerization. The mixture was poured into a large quantity of methanol $(50 \mathrm{~mL})$. The obtained white polymer was filtered and then dried under vacuum at $40{ }^{\circ} \mathrm{C}$ to a constant weight. The block copolymer was obtained in a yield $85 \%$. The block product was purified by hot hexane extraction for $6 \mathrm{~h}$ with a little of BHT.

Computational details. All calculations were performed with the Gaussian 09 program. ${ }^{2}$ The B3PW91 hybrid exchange-correlation functional was utilized for geometry optimization. ${ }^{3-5}$ Each optimized structure was subsequently analyzed by harmonic vibration frequencies for characterization of a minimum (Nimag $=0$ ) or a transition state $($ Nimag $=1)$ and providing thermodynamic data. The transition state structures are shown to connect the reactant and product on either side via intrinsic reaction coordinate (IRC) following. The 6-31G* basis set was considered for $\mathrm{C}, \mathrm{H}$, and $\mathrm{N}$ atoms, and the $\mathrm{Si}, \mathrm{P}, \mathrm{Y}$, and $\mathrm{Lu}$ atoms were treated by the Stuttgart/Dresden effective core potential (ECP) and the associated basis sets. ${ }^{6}$ The basis sets of $\mathrm{Si}$ and $\mathrm{P}$ were augmented with one d-polarization function (exponent of 0.284 and 0.387 , respectively). ${ }^{7}$ This basis set is denoted as "BSI". To obtain more reliable relative energies, the single-point calculations of optimized structures were carried out at the level of B3PW91-D3 (B3PW91 with Grimme's DFT-D3 correction) ${ }^{8,9}$ BSII, taking into account solvation effect of toluene with the $\mathrm{SMD}^{10}$ solvation model. In the BSII, the $6-31+\mathrm{G}(\mathrm{d}, \mathrm{p})$ basis set was used for nonmetal atoms, while the basis sets together with associated pseudo potentials for $\mathrm{Y}$ and $\mathrm{Lu}$ atoms are the same as that in geometry optimization. Therefore, unless otherwise mentioned, the free energy $(\Delta \mathrm{G}, 298.15 \mathrm{~K}, 1 \mathrm{~atm})$ in solution, which was used for description of energy profiles, was obtained from the solvation single-point calculation and the gas-phase Gibbs free energy correction. The 3D molecular structures displayed in this paper were drawn by using CYLview. ${ }^{11}$ 
Mechanism of 1PB polymerization catalyzed by complex 8. Syndio 3,4-polymerization of 1PB by 8 is studied by DFT calculation. The optimized cationic active species cats $^{12}$ and the four coordination-insertion manners are considered as described for those of $\mathbf{3 b}$ (Figure S39). The first 1PB trans-re coordination to cat8 affords 8-P1 $\mathbf{1}_{\text {trans-re }}$ with a barrier of $9.9 \mathrm{kcal} \mathrm{mol}^{-1}$. This is slightly different from the initial step in cat3b where the first $1 \mathrm{~PB}$ coordination-insertion in the electronically prefered cis-re mode. This is probably because cat8 contains a bulky ancillary ligand, which arouses repulsion between the two phenyl groups in the ligand and 1PB to weaken the electronic superiority of cis-re mode. The non covalent interaction analyses ${ }^{13}$ of $\mathbf{8}-\mathbf{T S 1}_{\text {trans-re }}$ indicates the $\mathrm{C}-\mathrm{H} \cdot \boldsymbol{\bullet} \pi$ interaction between the two phenyl groups brings about more stronger interaction (Figure S42). Therefore, the first trans-re $1 \mathrm{~PB}$ insertion is a kinetically prefered pathway. ${ }^{14} \mathbf{8}-\mathbf{P} \mathbf{1}_{\text {trans-re }}$ accepts the second trans-si $1 \mathrm{~PB}$ via the transition state 8-TS2 trans-si-34 $_{\text {to }}$ to afford the most stable intermediate $\mathbf{8}-\mathbf{P} \mathbf{2}_{\text {trans-si-34 }}\left(-43.9 \mathrm{kcal} \mathrm{mol}^{-1}\right)$ by acrossing the lowest barrier $\left(13.3 \mathrm{kcal} \mathrm{mol}^{-1}\right)$ and to give the first syndiotactic 3,4-poly(1PB) unit. This is also in agreement with the geometry and energy analyses (Figure S42, Table S6) that the transition state 8-TS2 trans-si-34 $_{\text {holds the stronger }}$ interaction $\left(-57.0 \mathrm{kcal} \mathrm{mol}^{-1}\right)$ and the smaller deformation $\left(51.3 \mathrm{kcal} \mathrm{mol}^{-1}\right)$, suggesting a kinetically prefered pathway. On the contrary, the 1,4-insertion of 1PB into 8 overcomes a bigger deformation energy to destroy the conjugation between allyl $(\mathrm{C} 1-\mathrm{C} 2-\mathrm{C} 3)$ and phenyl. This is probably the reason behind that a highly cis-1,4 selective catalyst $\mathbf{8}$ for diene polymerization provides 3,4-regioselectivity for 1PB polymerization.

\section{Reference:}

(1) (a) B. Liu, L. Li, G. Sun, J. Liu, M. Wang, S. Li, D. Cui, 3,4-polymerization of isoprene by using NSN- and NPN-ligated rare earth metal precursors: switching of stereo selectivity and mechanism. Macromolecules 2014, 47, 4971-4978; (b) S. Li, D. Cui, D. Li, Z. Hou, Highly 3,4-selective polymerization of isoprene with NPN ligand stabilized rare-earth metal bis(alkyl)s. structures and performances. Organometallics 2009, 28, 4814-4822. (c) Y. Pan, W. Rong, Z. Jian, D. Cui, Ligands dominate highly syndioselective polymerization of styrene by using constrained-geometry-configuration rare-earth metal precursors. Macromolecules 2012, 45, 1248-1253. (d) L. Zhang, T. Suzuki, Y. Luo, M. Nishiura, Z. Hou, Cationic alkyl rare-earth metal complexes bearing an ancillary bis(phosphinophenyl)amido ligand: acatalytic system for living cis1,4-polymerization and copolymerization of isoprene and butadiene. Angew. Chem. Int. Ed. 2007, 46, 1909-1913.

(2) M. Frisch, G. Trucks, H. Schlegel, G. Scuseria, M. Robb, J. heeseman, G. Scalmani, V. Barone, B. Mennucci, G. Petersson, H. Nakatsuji, M. Caricato, X. Li, H. Hratchian, A. Izmaylov, J. Bloino, G. Zheng, J. Sonnenberg, M. Hada, M. Ehara, K. Toyota, R. Fukuda, J. Hasegawa, M. Ishida, T. Nakajima, Y. Honda, O. Kitao, H. Nakai, T. Vreven, Jr. Montgomery, J. Peralta, F. Ogliaro, M. Bearpark, J. Heyd, E. Brothers, K. Kudin, V. Staroverov, R. Kobayashi, J. Normand, K. Raghavachari, A. Rendell, J. Burant, S. Iyengar, J. Tomasi, M. Cossi, N. Rega, N. Milam, M. Klene, J. Knox, J. Cross, V. Bskken, C. Adamo, J. Jaramillo, R. Gomperts, R. Stratmann, O. Yazyev, A. Austin, R. Cammi, C. Pomelli, J. Ochterski, R. Marin, K. Morokuma, V. Zakrzewski, G. Voth, G. Salvador, J. Dannenberg, S. Dapprich, A. Daniels, Ö. Farkas, J. Foresman, J. Ortiz, J. Cioslowski, D. Fox, Gaussian 09, Revision A.02, Gaussian, Inc., Wallingford, CT. 2009.

(3) A. Becke., Density-functional thermochemistry. III. The role of exact exchange. J. Chem. Phys. 1993, 98, 56485653.

(4) C. Lee, W. Yang, R. Parr, Development of the Colle-Salvetti correlation-energy formula into a functional of the electron density. Phys. Rev. B 1988, 37, 785-789.

(5) J. Perdew, K. Burke, Y. Wang, Generalized gradient approximation for the exchange-correlation hole of a many-electron system.Phys. Rev. B 1996, 54, 16533-16539.

(6) D. Andrae, H. U., M. Dolg, H. Stoll, Energy-adjustedab initio pseudopotentials for the second and third row transition elements. Theor. Chim. Acta 1990, 77, 123-141.

(7) A. Höllwarth, M. Böhme, S. Dapprich, A. W. Ehlers, A. Gobbi, V. Jonas, K. F. Köhler, R. Stegmann, A. Veldkamp, G. Frenking, A set of d-polarization functions for pseudo-potential basis sets of the main group elements $\mathrm{Al} \mathrm{H}$. Nakatsuji, M. Caricato, X. Li, H. Hratchian, A. IzChem. Phys. Lett. 1993, 208, 237-240. 
(8) S. Grimme, J. Antony, S. Ehrlich, H. Krieg, A consistent and accurate ab initio parametrization of density functional dispersion correction (DFT-D) for the 94 elements H-Pu., J. Chem. Phys. 2010, 132, 154104-15424.

(9) Stefan. Grimme, Accurate description of van der Waals complexes by density functional theory including empirical corrections. J. Comput. Chem. 2004, 25, 1463-1473.

(10) A. Marenich, C. Cramer, D. Truhlar, Universal solvation model based on solute electron density and on a continuum model of the solvent defined by the bulk dielectric constant and atomic surface tensions. J. Phys. Chem. B.

2009, 113, 6378-6396.

(11) C.Y. Lview, C. Y. Legault, Université de Sherbrooke, 2009 (http://www.cylview.org)

(12) Wang, X. B.; Kang, X. H.; Zhou, G. L.; Qu, J. P.; Hou, Z. M., Luo, Y. DFT studies on cis-1,4-polymerization of dienes catalyzed by a cationic rare-earth metal complex bearing an ancillary PNP ligand, Polymers 2017, 9, 53.

(13) (a) Lu, T.; Chen, F. Multiwfn: A multifunctional wavefunction analyzer, J. Comput. Chem., 2012, 33, 580-592; b) Lefebvre, C.; Rubez, G.;Khartabil, H.;Boisson, J.;García, J.;Hénon, E. Accurately extracting the signature of intermolecular interactions present in the NCI plot of the reduced density gradient versus electron density, Phys. Chem. Chem. Phys., 2017, 19, 17928-17936; c) Humphrey, W.;Dalke, A.;Schulten, K.VMD: Visual molecular dynamics, J. Mol. Graphics 1996, 14, 33-38.

(14) A energy decomposition analysis (Table S5) further confirmed that a slight preference for trans-re insertion was mainly origined from a bigger interaction energy ( -23.9 vs. -22.4 (trans-si) and -23.1 (cis-si) $\mathrm{kcal} \mathrm{mol}^{-1}$ ) and a smaller deformation energy (28.7 vs. 29.4 (trans-si), 28.9 (cis-si), and 35.5 (cis-re) kcal mol-1), which is consistent with the previous geometric and interaction analysis.

Table S1. Polymerization of 1PB with rare-earth metal complexes.

\begin{tabular}{|c|c|c|c|c|c|c|c|c|}
\hline $\operatorname{Run}^{[\mathrm{a}]}$ & Cat. & $\begin{array}{c}\text { Temp. } \\
\left({ }^{\circ} \mathrm{C}\right)\end{array}$ & $\begin{array}{l}\text { Time } \\
(\min )\end{array}$ & $\begin{array}{c}\text { Yield } \\
(\%)\end{array}$ & $\begin{array}{c}3,4-[b] \\
(\%)\end{array}$ & $\begin{array}{c}r r r r^{[b]} \\
(\%)\end{array}$ & $\begin{array}{l}M_{\mathrm{n}}[\mathrm{c}] \\
\left(10^{4}\right)\end{array}$ & $M_{\mathrm{n}} / M_{\mathrm{w}}[\mathrm{c}]$ \\
\hline 1 & $4 b$ & 0 & 60 & 85 & $>99$ & 97.1 & 2.40 & 1.73 \\
\hline 2 & $4 b$ & 40 & 20 & 98 & $>99$ & 98.2 & 2.20 & 1.73 \\
\hline 3 & $4 b$ & 60 & 20 & 100 & $>99$ & 97.1 & 1.25 & 1.95 \\
\hline $4^{[\mathrm{d}]}$ & $4 b$ & 25 & 60 & 98 & $>99$ & 97.3 & 1.15 & 1.86 \\
\hline $5^{[\mathrm{e}]}$ & $4 b$ & 25 & 60 & 20 & $>99$ & 97.3 & $\begin{array}{l}1.82 / \\
0.23\end{array}$ & $\begin{array}{l}1.70 / \\
1.32\end{array}$ \\
\hline $6^{[\mathrm{f}]}$ & $4 b$ & 25 & 60 & 93 & $>99$ & 98.0 & 1.86 & 1.72 \\
\hline $7^{[\mathrm{f}]}$ & $3 b$ & 25 & 60 & 75 & 98.1 & 93.9 & 2.77 & 1.67 \\
\hline $8^{[\mathrm{f}]}$ & 1 & 25 & 60 & trace & - & - & - & - \\
\hline
\end{tabular}

[a] Conditions: cat. $10 \mu \mathrm{mol} ;\left[\mathrm{Ph}_{3} \mathrm{C}\right]\left[\mathrm{B}\left(\mathrm{C}_{6} \mathrm{~F}_{5}\right)_{4}\right], 10 \mu \mathrm{mol}$; $\mathrm{Al}^{i} \mathrm{Bu}_{3} 100 \mu \mathrm{mol},[(\mathrm{E})-1-\mathrm{PB}]=$ $1.5 \mathrm{~mol} / \mathrm{mL}$. [b] Determined by ${ }^{1} \mathrm{H}$ NMR and ${ }^{13} \mathrm{C}$ NMR spectroscopy in $\mathrm{CDCl}_{3}$. [c] Determined by GPC in THF at $40{ }^{\circ} \mathrm{C}$ against polystyrene standard. [d] $\mathrm{AlEt}_{3}, 100 \mu \mathrm{mol}$. [e] $\mathrm{AlMe}_{3}, 100 \mu \mathrm{mol}$. [f] [B( $\left.\mathrm{C}_{6} \mathrm{~F}_{5}\right)_{3} 10 \mu \mathrm{mol}$. 
Table S2. Polymerization of 1PB with lutetium precursor $4 \mathbf{b}$.

\begin{tabular}{cccccccc}
\hline Run $^{[\mathrm{a}]}$ & {$[\mathbf{4 b}]:[1 \mathrm{~PB}]$} & $\begin{array}{c}\text { Tol. } \\
(\mathrm{mL})\end{array}$ & $\begin{array}{c}\text { Time } \\
(\mathrm{min})\end{array}$ & $\begin{array}{c}\text { Yield } \\
(\%)\end{array}$ & $\begin{array}{c}3,4-[\mathrm{b}] \\
(\%)\end{array}$ & $\begin{array}{c}M_{\mathrm{n}}{ }^{[\mathrm{c}]} \\
\left(10^{4}\right)\end{array}$ & $M_{\mathrm{w}} / M_{\mathrm{n}}{ }^{[\mathrm{c}]}$ \\
\hline 1 & $300: 1$ & 2 & 40 & 74 & $>99$ & 4.91 & 1.71 \\
2 & $450: 1$ & 3 & 60 & 83 & $>99$ & 5.31 & 1.76 \\
3 & $600: 1$ & 4 & 90 & 79 & $>99$ & 8.05 & 1.69 \\
4 & $750: 1$ & 5 & 150 & 84 & $>99$ & 10.2 & 1.67 \\
\hline
\end{tabular}

[a] Conditions: cat. $10 \mu \mathrm{mol} ;\left[\mathrm{Ph}_{3} \mathrm{C}\right]\left[\mathrm{B}\left(\mathrm{C}_{6} \mathrm{~F}_{5}\right)_{4}\right], 10 \mu \mathrm{mol} ; \mathrm{Al}^{i} \mathrm{Bu}_{3} 100 \mu \mathrm{mol},[(\mathrm{E})-1-\mathrm{PB}]=1.5$ $\mathrm{mol} / \mathrm{mL}$. [b] Determined by ${ }^{1} \mathrm{H}$ NMR and ${ }^{13} \mathrm{C}$ NMR spectroscopy in $\mathrm{CDCl}_{3}$. [c] Determined by $\mathrm{GPC}$ in $\mathrm{THF}$ at $40^{\circ} \mathrm{C}$ against polystyrene standard.

Table S3. Energy $\left(\mathrm{kcal} \mathrm{mol}^{-1}\right)$ decomposition analysis of transition states in chain initiation steps by species cat3b.

\begin{tabular}{lccccc}
$\begin{array}{c}\text { Insertion } \\
\text { fashions }\end{array}$ & $\Delta E_{\text {int }}$ & $\Delta E_{\text {def }}(\mathbf{c a t} \mathbf{3 b})$ & $\Delta E_{\text {def }}(\mathbf{1 P B})$ & $\Delta E_{\text {def }}$ & $\Delta E_{\mathrm{TS}}$ \\
\hline 3b-TS1 $_{\text {trans-re }}$ & -49.2 & 26.7 & 10.2 & 36.9 & -12.3 \\
3b-TS1 $_{\text {trans-si }}$ & -45.5 & 27.8 & 9.6 & 37.4 & -8.1 \\
3b-TS1 $_{\text {cis-re }}$ & -52.1 & 25.8 & 12.2 & 38.0 & -14.1 \\
3b-TS1 $_{\text {cis-si }}$ & -49.4 & 26.2 & 11.9 & 38.1 & -11.3 \\
\hline
\end{tabular}

Table S4. Energy $\left(\mathrm{kcal} \mathrm{mol}^{-1}\right)$ decomposition analysis of transition states in chain propagation processes by species cat3b.

\begin{tabular}{lccccc}
\hline $\begin{array}{l}\text { Insertion } \\
\text { fashions }\end{array}$ & $\Delta E_{\text {int }}$ & $\begin{array}{c}\Delta E_{\text {def }}\left(\mathbf{3 b}_{\text {rans-si }}\right) \\
\text { rat }\end{array}$ & $\Delta E_{\text {def }}(\mathbf{1 P B})$ & $\Delta E_{\text {def }}$ & $\Delta E_{\mathrm{TS}}$ \\
\hline $\mathbf{3 b - T S 3}_{\text {trans-re }}$ & -55.1 & 45.4 & 11.8 & 57.2 & 2.1 \\
$\mathbf{3 b - T S 3}_{\text {trans-si }}$ & -52.5 & 53.2 & 13.3 & 66.5 & 14.1 \\
$\mathbf{3 b - T S 3}_{\text {cis-re }}$ & -62.0 & 49.8 & 15.1 & 64.9 & 2.9 \\
$\mathbf{3 b - T S 3}_{\text {cis-si }}$ & -55.6 & 54.6 & 15.7 & 70.4 & 14.8 \\
\hline
\end{tabular}

Table S5. Energy $\left(\mathrm{kcal} \mathrm{mol}^{-1}\right)$ decomposition analysis of transition states in chain initiation steps by species cat8 ${ }^{\text {a }}$.

\begin{tabular}{cccccc}
\hline $\begin{array}{c}\text { Insertion } \\
\text { fashions }\end{array}$ & $\Delta E_{\text {int }}$ & $\Delta E_{\text {def }}(\mathbf{c a t 8})$ & $\Delta E_{\text {def }}(\mathbf{P B})$ & $\Delta E_{\text {def }}$ & $\Delta E_{\mathrm{TS}}$ \\
\hline 8-TS1 $_{\text {trans-re }}$ & -23.9 & 19.9 & 8.8 & 28.7 & 4.8 \\
8-TS1 1 trans-si $_{\text {i }}$ & -22.4 & 21.0 & 8.4 & 29.4 & 7.0 \\
8-TS1 $_{\text {cis-re }}$ & -26.8 & 26.9 & 8.6 & 35.5 & 8.7 \\
8-TS1 $_{\text {cis-si }}$ & -23.1 & 19.6 & 9.3 & 28.9 & 5.8 \\
\hline
\end{tabular}

${ }^{\mathrm{a}}$ To get the reasons behind, energy decomposition analyses on the transition states 8-TS1 ${ }_{\text {trans-re }}$, 8-TS1 $1_{\text {trans-si }}$, 8-TS1 cis-re $_{\text {, }}$ and 8-TS1 cis-si $_{\text {. }}$ were carried out. The energy of 8-TS1, $\Delta E_{\mathrm{TS}}$, is evaluated by the equation $\Delta E_{\mathrm{TS}}=\Delta E_{\text {int }}+\Delta E_{\text {def }}$ (cat8) $+\Delta E_{\text {def }}(\mathbf{1 P B})$. $\Delta E_{\text {int }}$ represents the interaction energy between the two fragments in 8-TS1: the monomer unit 1 PB and the remained yttrium active species cat8. $\Delta E_{\text {def }}($ cat8) and $\Delta E_{\text {def }}(\mathbf{P B})$ are the deformation energies of the two fragments in 8-TS1 compared to their optimal geometries.

Table S6. Energy $\left(\mathrm{kcal} \mathrm{mol}^{-1}\right)$ decomposition analysis of transition states in chain propagation processes by species cat8. 


\begin{tabular}{cccccc}
\hline $\begin{array}{c}\text { Insertion } \\
\text { fashions }\end{array}$ & $\Delta E_{\text {int }}$ & $\begin{array}{c}\Delta E_{\text {def }}(\mathbf{8}-\mathbf{P} \\
\left.\mathbf{1}_{\text {trans-re }}\right)\end{array}$ & $\Delta E_{\text {def }}(\mathbf{1 P B})$ & $\Delta E_{\text {def }}$ & $\Delta E_{\mathrm{TS}}$ \\
\hline $\mathbf{8 - T S 2}_{\text {trans-re-3,4 }}$ & -53.3 & 34.8 & 15.4 & 50.2 & -3.1 \\
$\mathbf{8 - T S 2}_{\text {trans-re-1,4 }}$ & -59.1 & 36.6 & 20.5 & 57.1 & -2.0 \\
8-TS2 $_{\text {trans-si-3,4 }}$ & -57.0 & 36.1 & 15.2 & 51.3 & -5.7 \\
8-TS2 $_{\text {trans-si-1,4 }}$ & -60.2 & 39.0 & 17.0 & 56.0 & -4.2 \\
\hline
\end{tabular}

Table S7. Energy $\left(\mathrm{kcal} \mathrm{mol}^{-1}\right)$ decomposition analysis of transition states in chain propagation processes by species cat7 and cat8.

\begin{tabular}{lccccc}
\hline $\begin{array}{c}\text { Insertion } \\
\text { fashions }\end{array}$ & $\Delta \mathrm{E}_{\text {int }}$ & $\Delta \mathrm{E}_{\text {def }}\left(\mathbf{8} / \mathbf{7 - P} \mathbf{1}_{\text {trans- } r e}\right)$ & $\Delta \mathrm{E}_{\text {def }}(1 \mathrm{~PB})$ & $\Delta \mathrm{E}_{\text {def }}$ & $\Delta \mathrm{E}_{\mathrm{TS}}$ \\
\hline 8-TS2 $_{\text {trans-re-3,4 }}$ & -53.3 & 34.8 & 15.4 & 50.2 & -3.1 \\
8-TS2 $_{\text {trans-re-1,4 }}$ & -59.1 & 36.6 & 20.5 & 57.1 & -2.0 \\
8-TS2 $_{\text {trans-si-3,4 }}$ & -57.0 & 36.1 & 15.2 & 51.3 & -5.7 \\
8-TS2 $_{\text {trans-si-1,4 }}$ & -60.2 & 39.0 & 17.0 & 56.0 & -4.2 \\
7-TS2 $_{\text {8-TS3 }}$ & -53.3 & 55.0 & 13.8 & 68.8 & 15.5 \\
7-TS3 & -49.6 & 40.1 & 13.3 & 53.4 & 3.8 \\
\hline
\end{tabular}

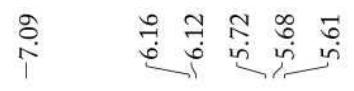
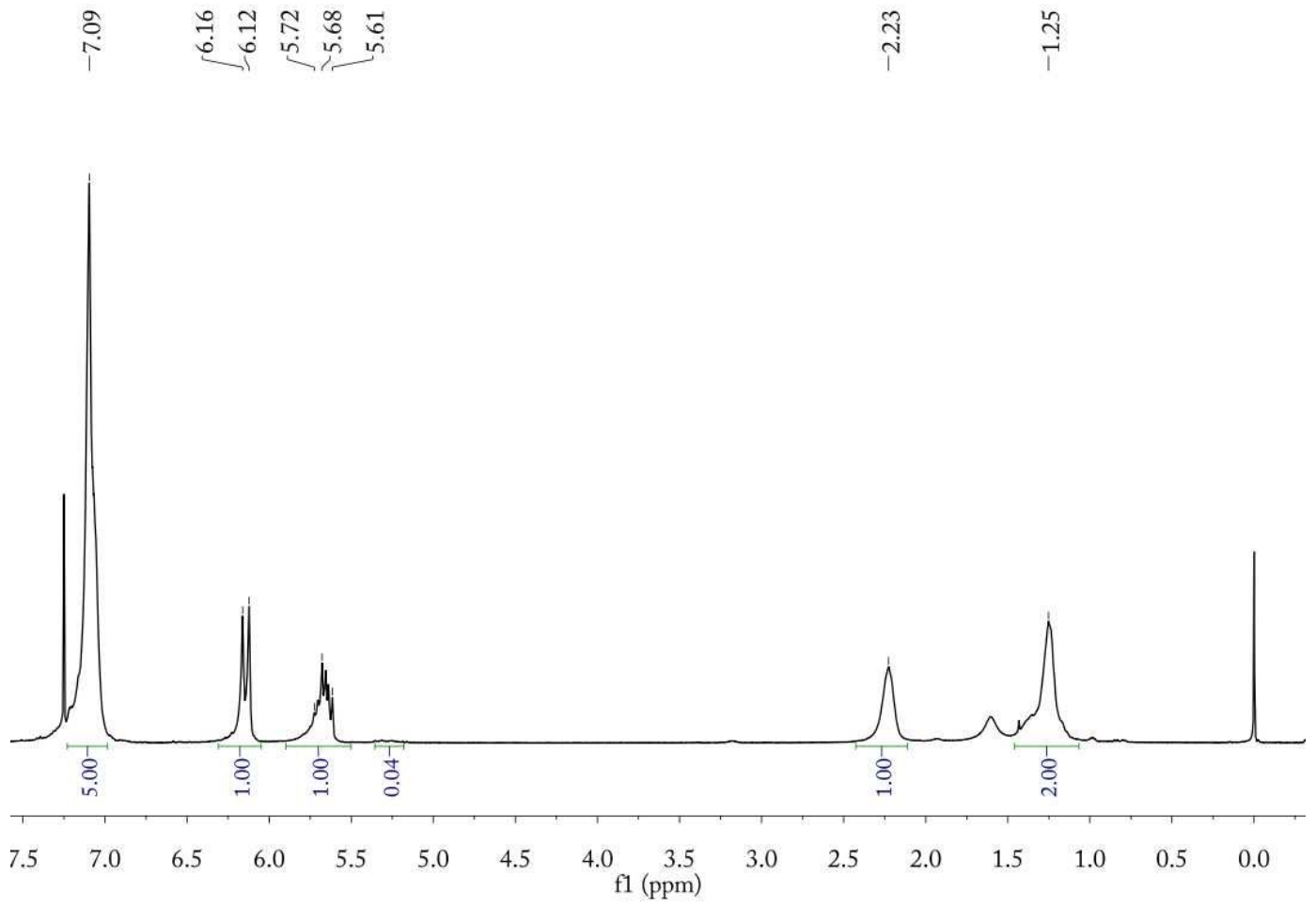

Figure S1. ${ }^{1} \mathrm{H}$ NMR $\left(400 \mathrm{~Hz}, 25^{\circ} \mathrm{C}, \mathrm{CDCl}_{3}\right)$ spectrum of 3,4-poly(1PB) (Table 1, run 1). 


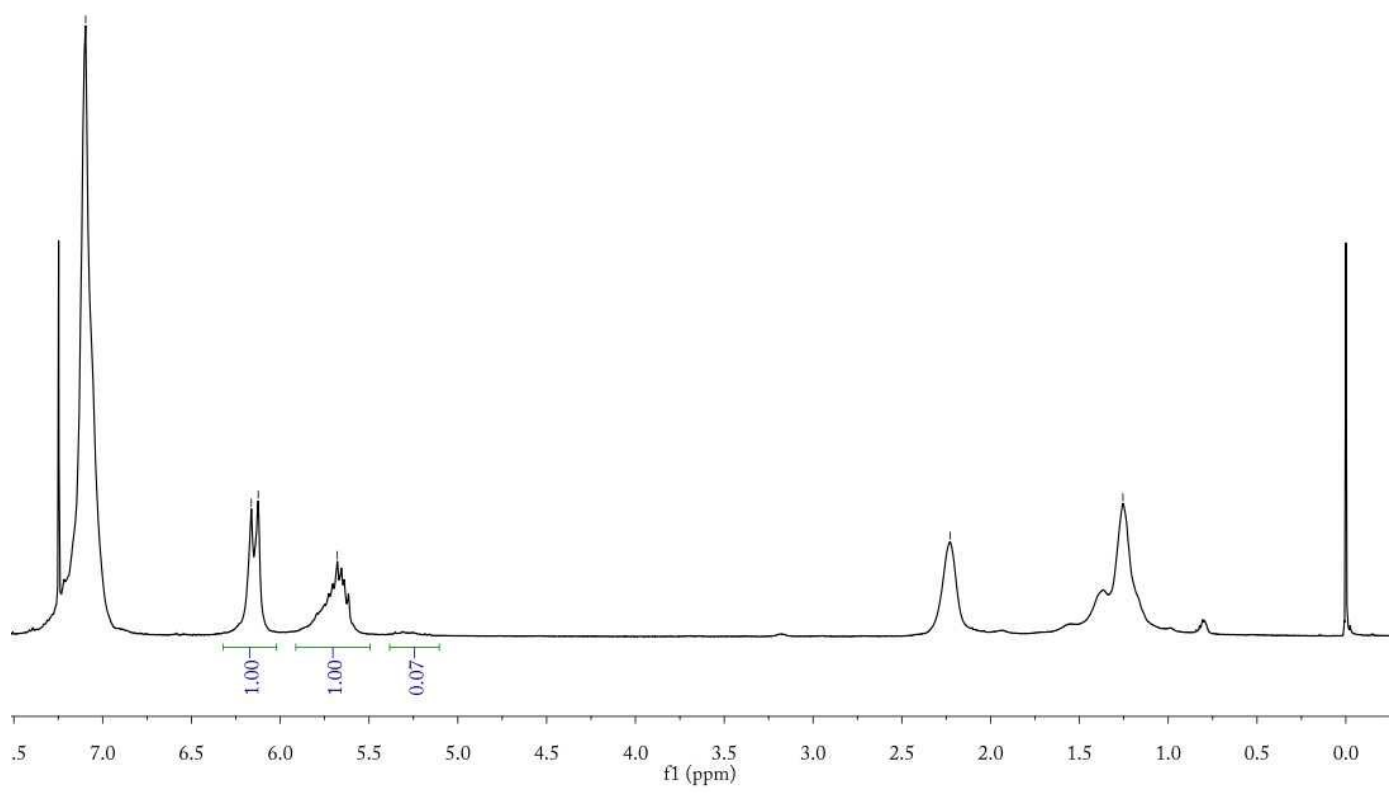

Figure S2. ${ }^{1} \mathrm{H}$ NMR $\left(400 \mathrm{~Hz}, 25^{\circ} \mathrm{C}, \mathrm{CDCl}_{3}\right)$ spectrum of 3,4-poly(1PB) (Table 1, run 2).

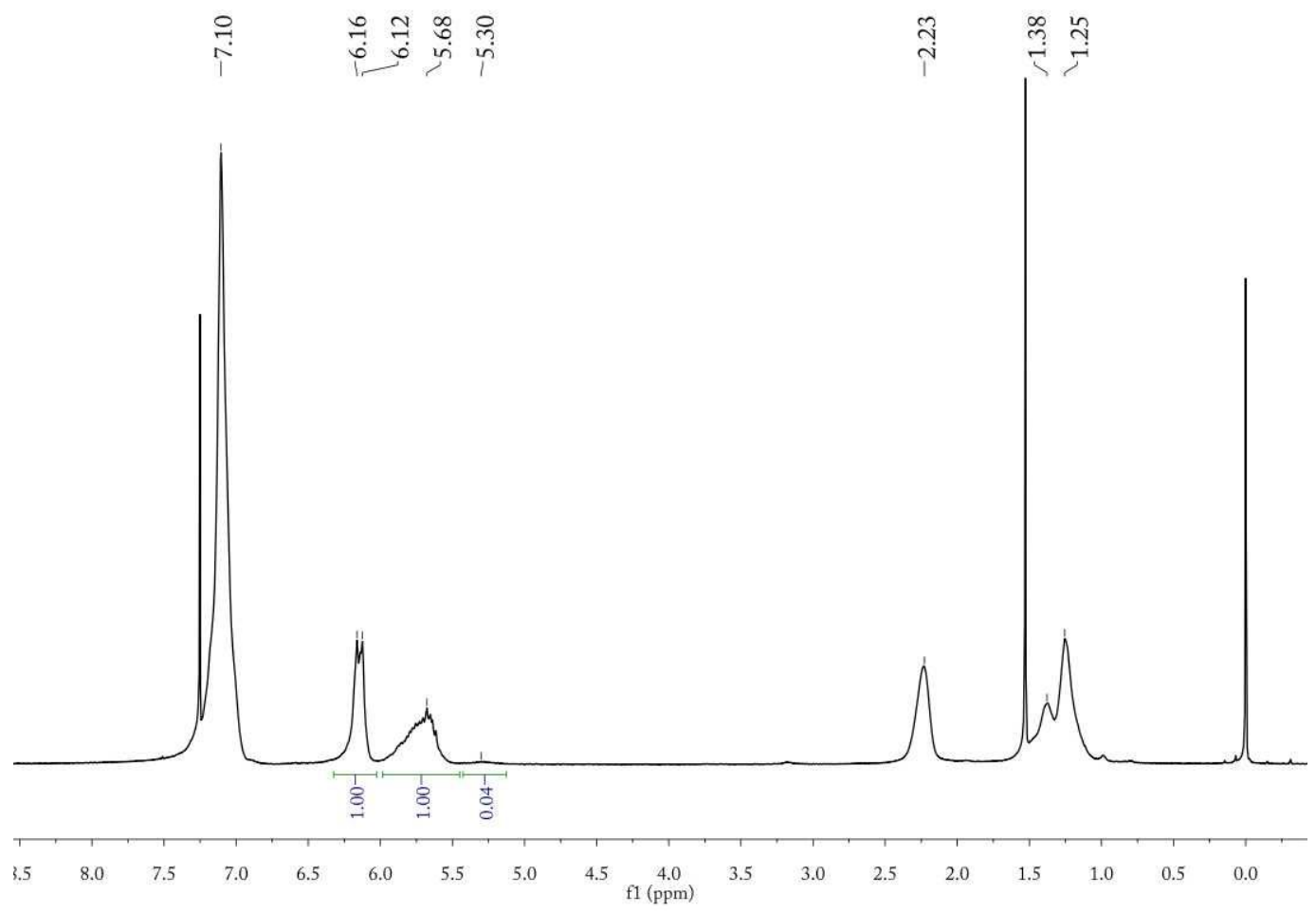

Figure S3. ${ }^{1} \mathrm{H}$ NMR $\left(400 \mathrm{~Hz}, 25^{\circ} \mathrm{C}, \mathrm{CDCl}_{3}\right)$ spectrum of 3,4-poly(1PB) (Table 1, run 3). 


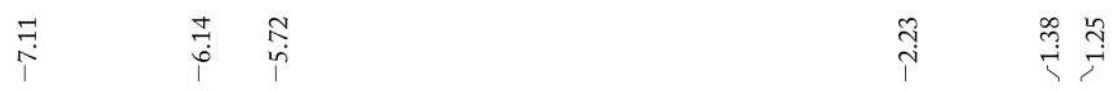

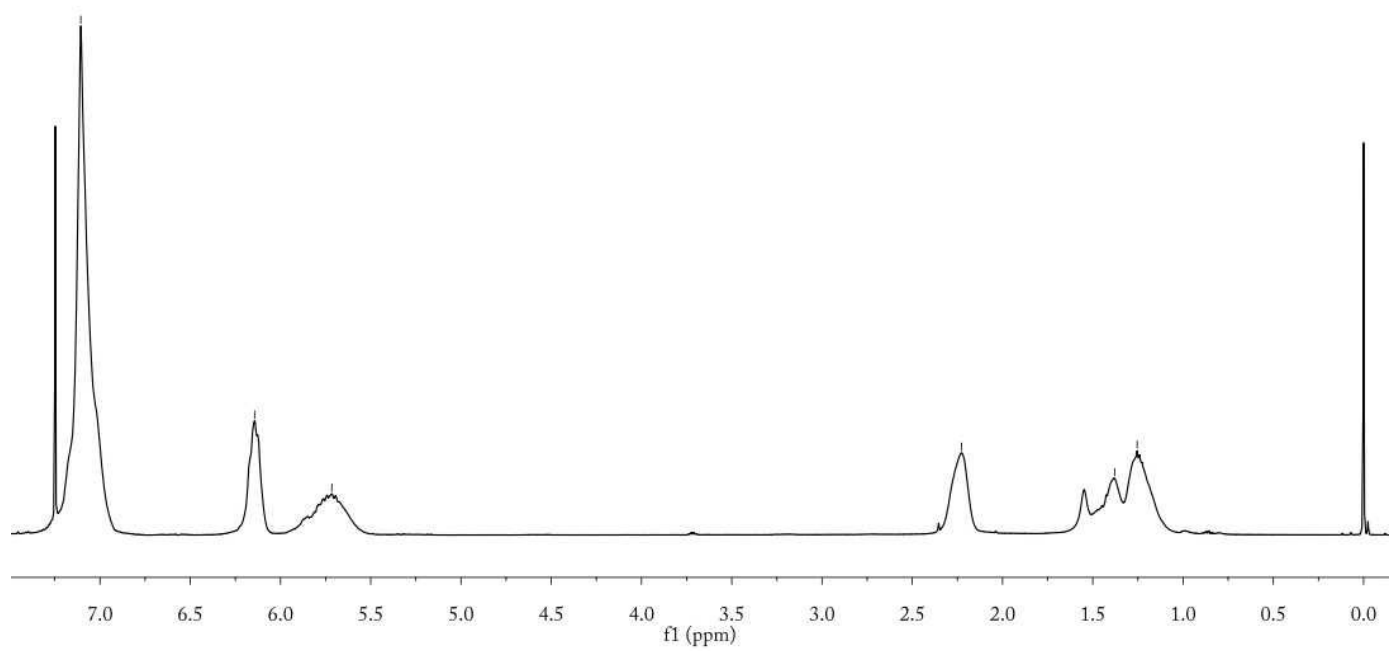

Figure S4. ${ }^{1} \mathrm{H}$ NMR $\left(400 \mathrm{~Hz}, 25^{\circ} \mathrm{C}, \mathrm{CDCl}_{3}\right)$ spectrum of 3,4-poly(1PB) (Table 1, run 4).

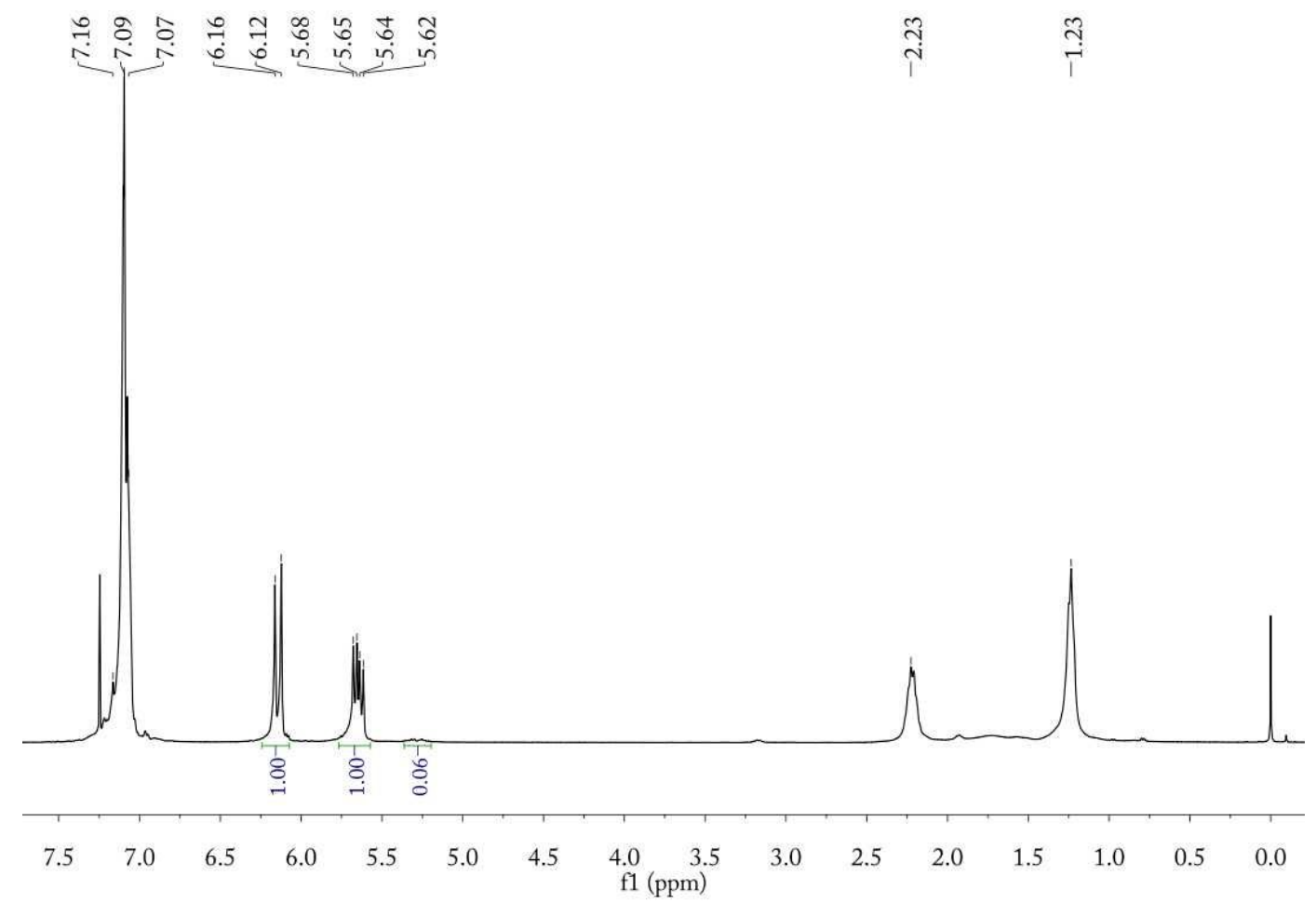

Figure S5. ${ }^{1} \mathrm{H}$ NMR $\left(400 \mathrm{~Hz}, 25^{\circ} \mathrm{C}, \mathrm{CDCl}_{3}\right)$ spectrum of 3,4-poly(1PB) (Table 1, run 5). 


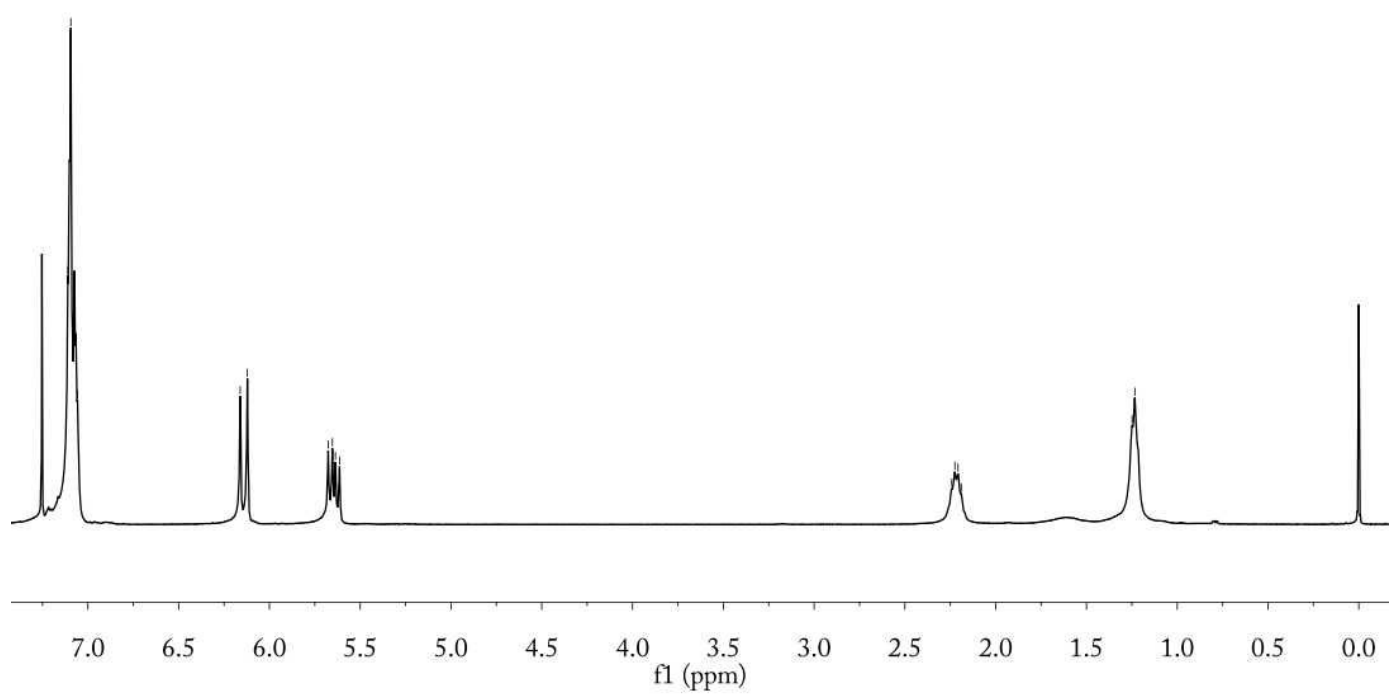

Figure S6. ${ }^{1} \mathrm{H}$ NMR $\left(400 \mathrm{~Hz}, 25^{\circ} \mathrm{C}, \mathrm{CDCl}_{3}\right)$ spectrum of 3,4-poly(1PB) (Table 1, run 6).

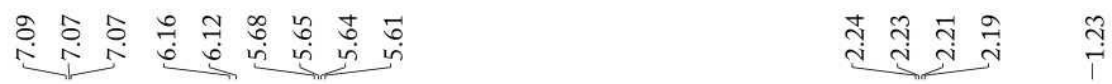

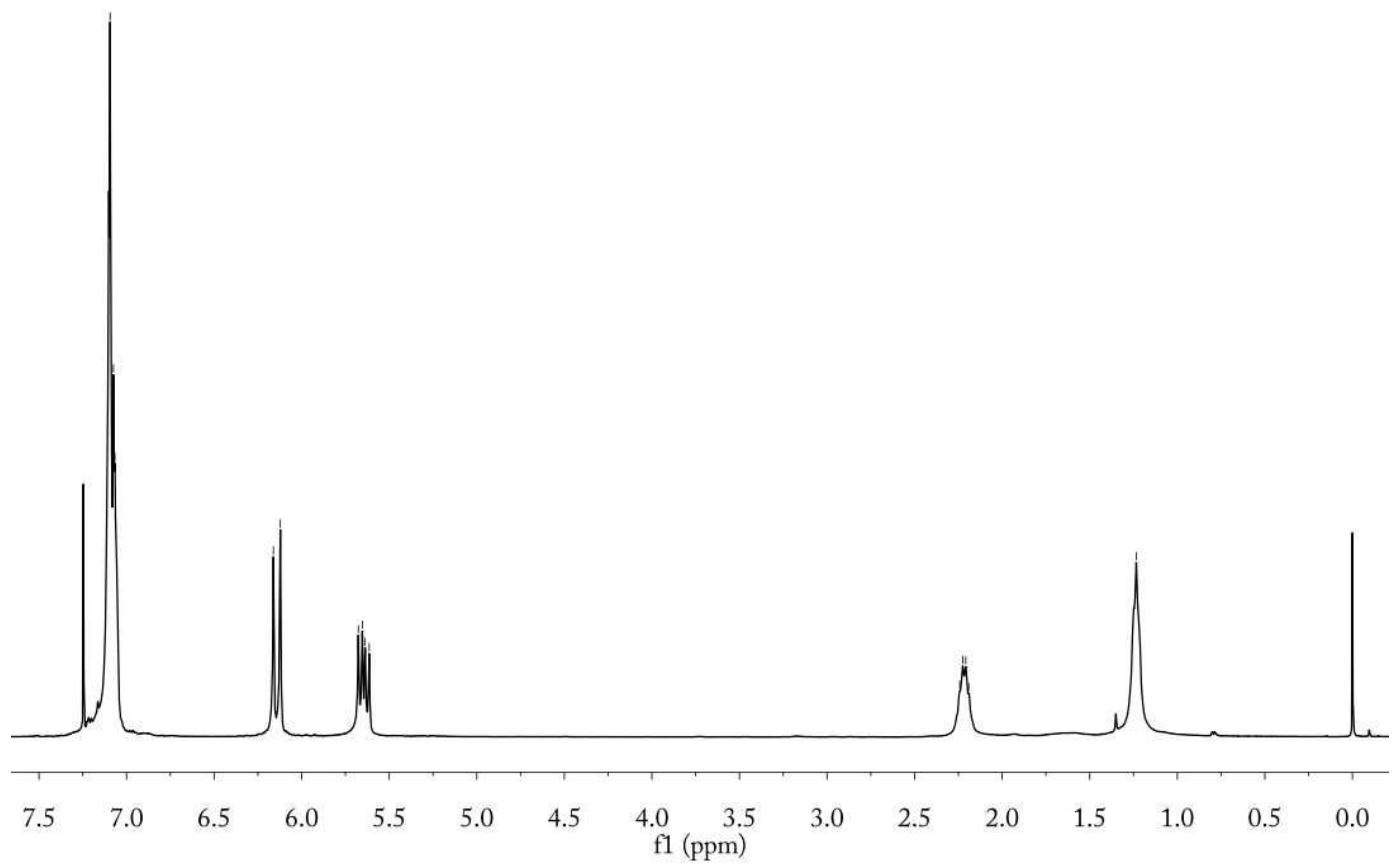

Figure S7. ${ }^{1} \mathrm{H}$ NMR $\left(400 \mathrm{~Hz}, 25^{\circ} \mathrm{C}, \mathrm{CDCl}_{3}\right)$ spectrum of 3,4-poly(1PB) (Table 1, run 8). 


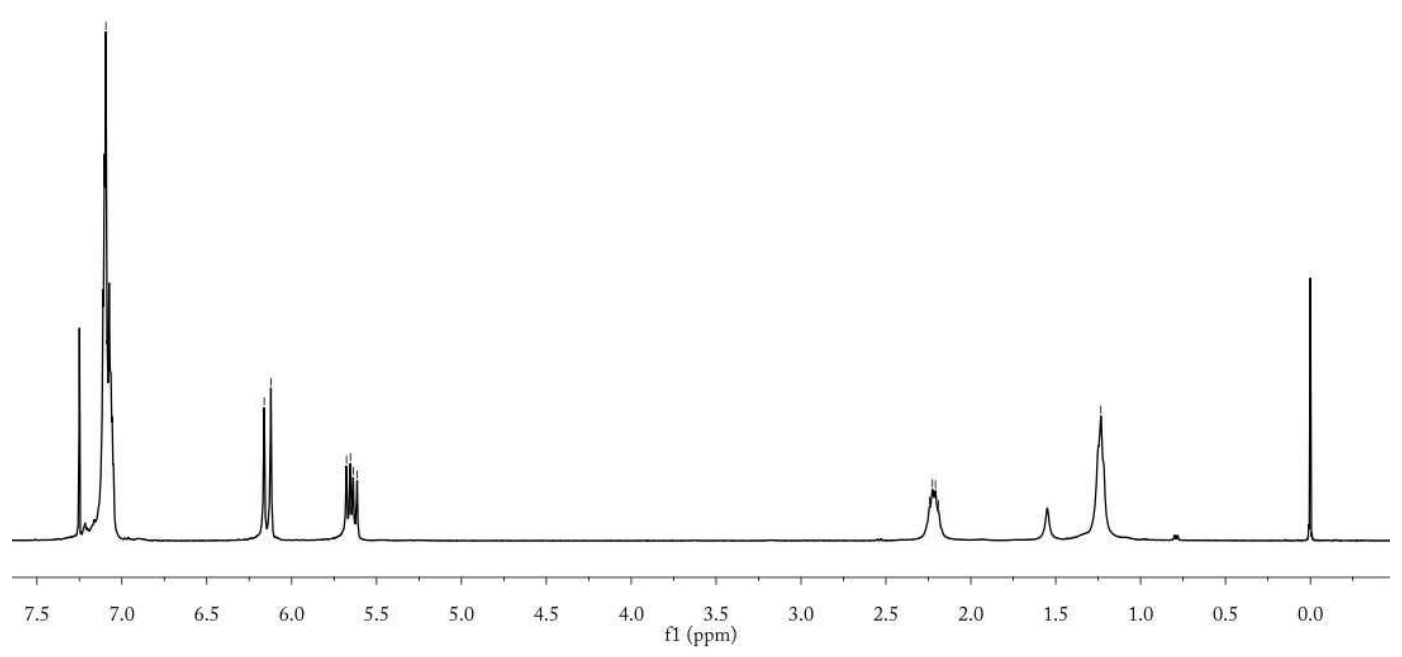

Figure S8. ${ }^{1} \mathrm{H} \mathrm{NMR}\left(400 \mathrm{~Hz}, 25^{\circ} \mathrm{C}, \mathrm{CDCl}_{3}\right)$ spectrum of 3,4-poly(1PB) (Table 1, run 9).

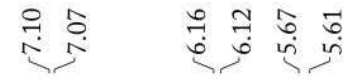
กิ

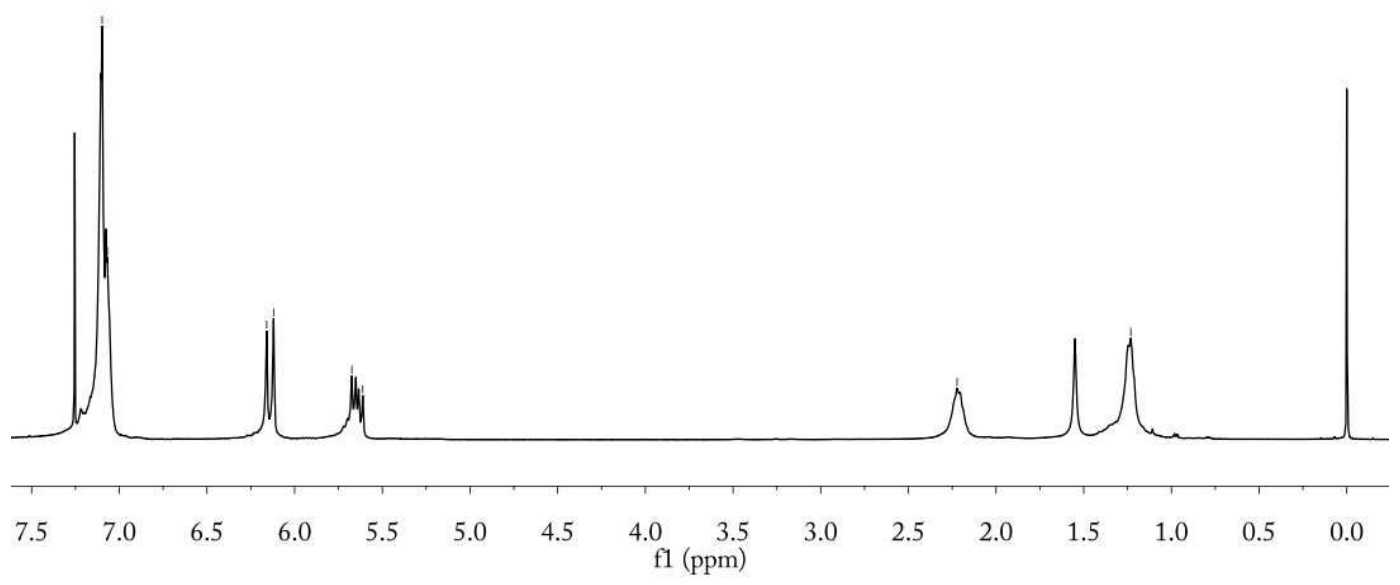

Figure S9. ${ }^{1} \mathrm{H}$ NMR $\left(400 \mathrm{~Hz}, 25^{\circ} \mathrm{C}, \mathrm{CDCl}_{3}\right)$ spectrum of 3,4-poly(1PB) (Table 1, run 15). 


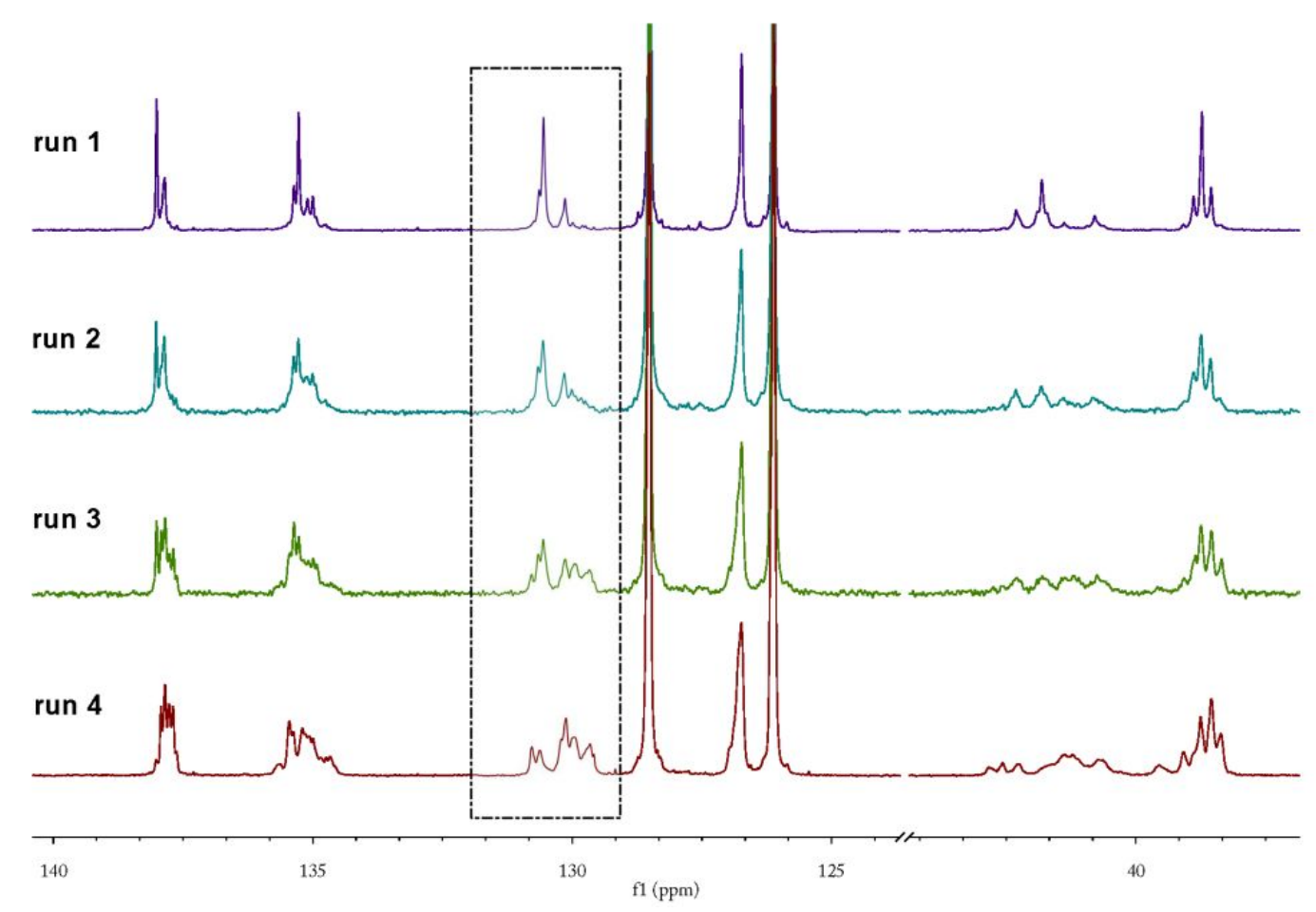

Figure S10. ${ }^{13} \mathrm{C}$ NMR $\left(100 \mathrm{~Hz}, 25^{\circ} \mathrm{C}, \mathrm{CDCl}_{3}\right)$ spectra of 3,4-poly(1PB) (Table 1, runs 1-4).

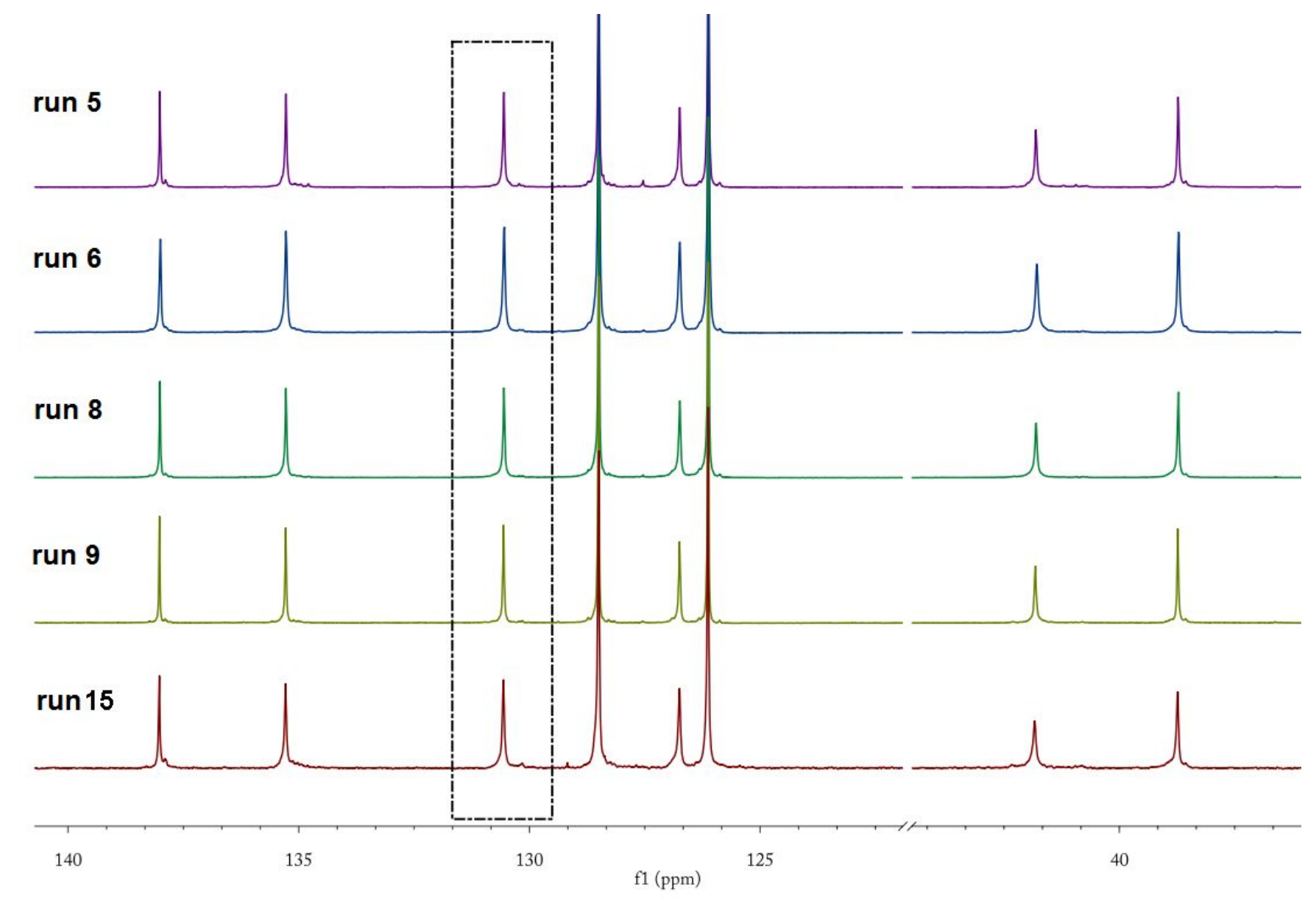

Figure S11. ${ }^{13} \mathrm{C}$ NMR $\left(100 \mathrm{~Hz}, 25{ }^{\circ} \mathrm{C}, \mathrm{CDCl}_{3}\right)$ spectra of 3,4-poly(1PB) (Table 1, runs 5,6,8,9,15). 


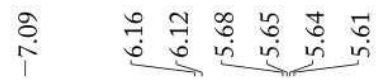

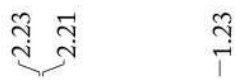

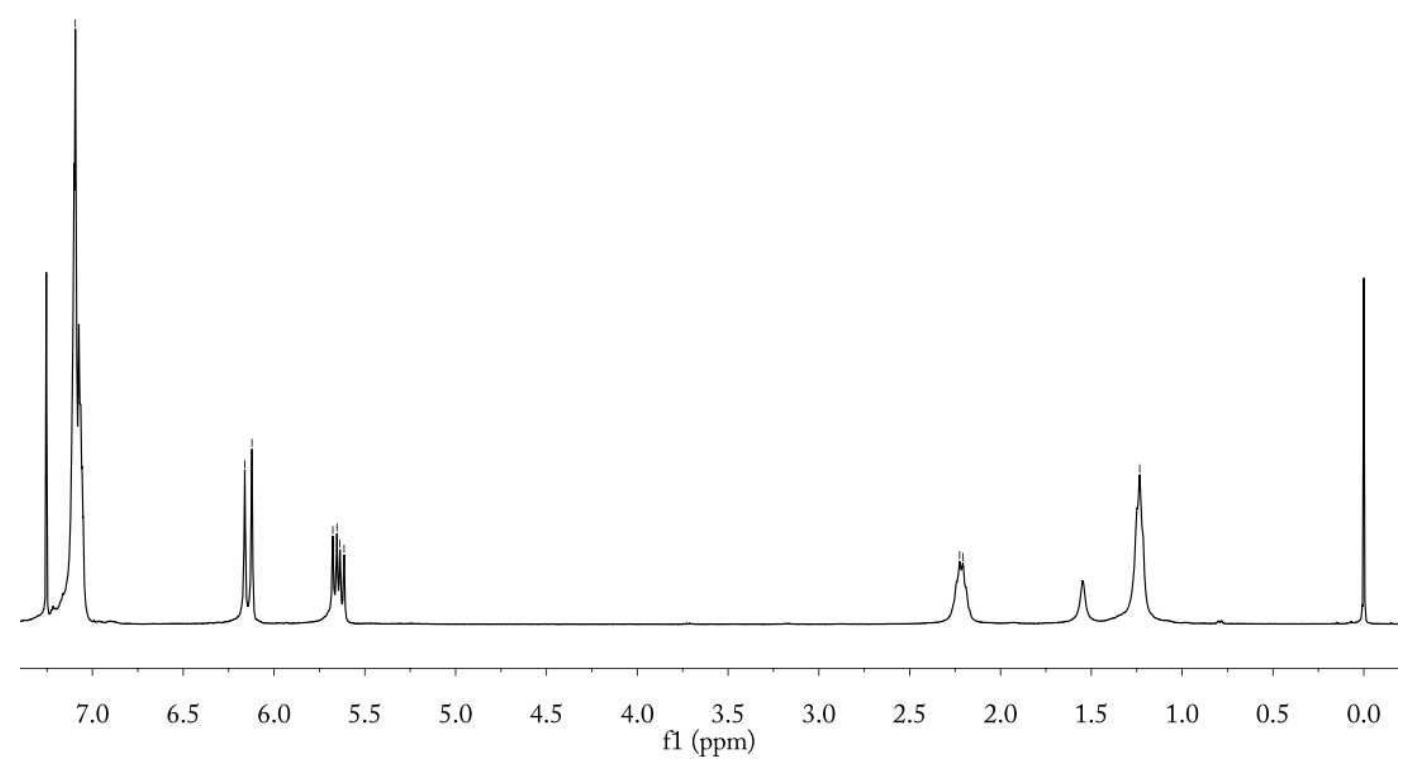

Figure S12. ${ }^{1} \mathrm{H}$ NMR $\left(400 \mathrm{~Hz}, 25^{\circ} \mathrm{C}, \mathrm{CDCl}_{3}\right)$ spectrum of 3, 4-poly(1PB) (Table S1, run 1).

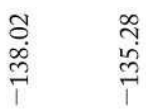

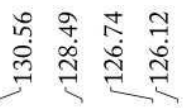

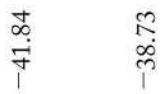

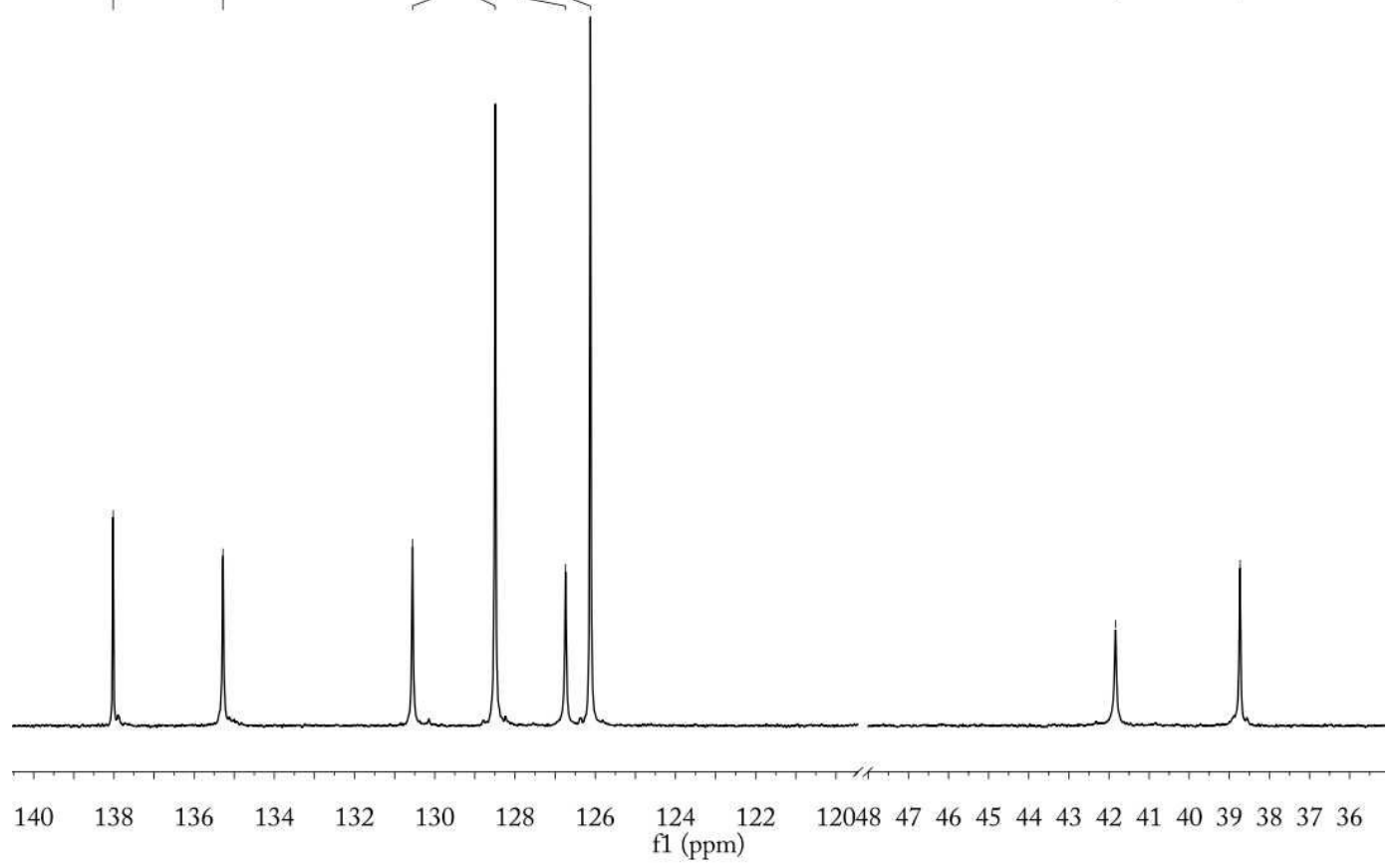

Figure S13 ${ }^{13} \mathrm{C}$ NMR $\left(100 \mathrm{~Hz}, 25{ }^{\circ} \mathrm{C}, \mathrm{CDCl}_{3}\right)$ spectrum of 3,4-poly(1PB) (Table S1, run 1). 


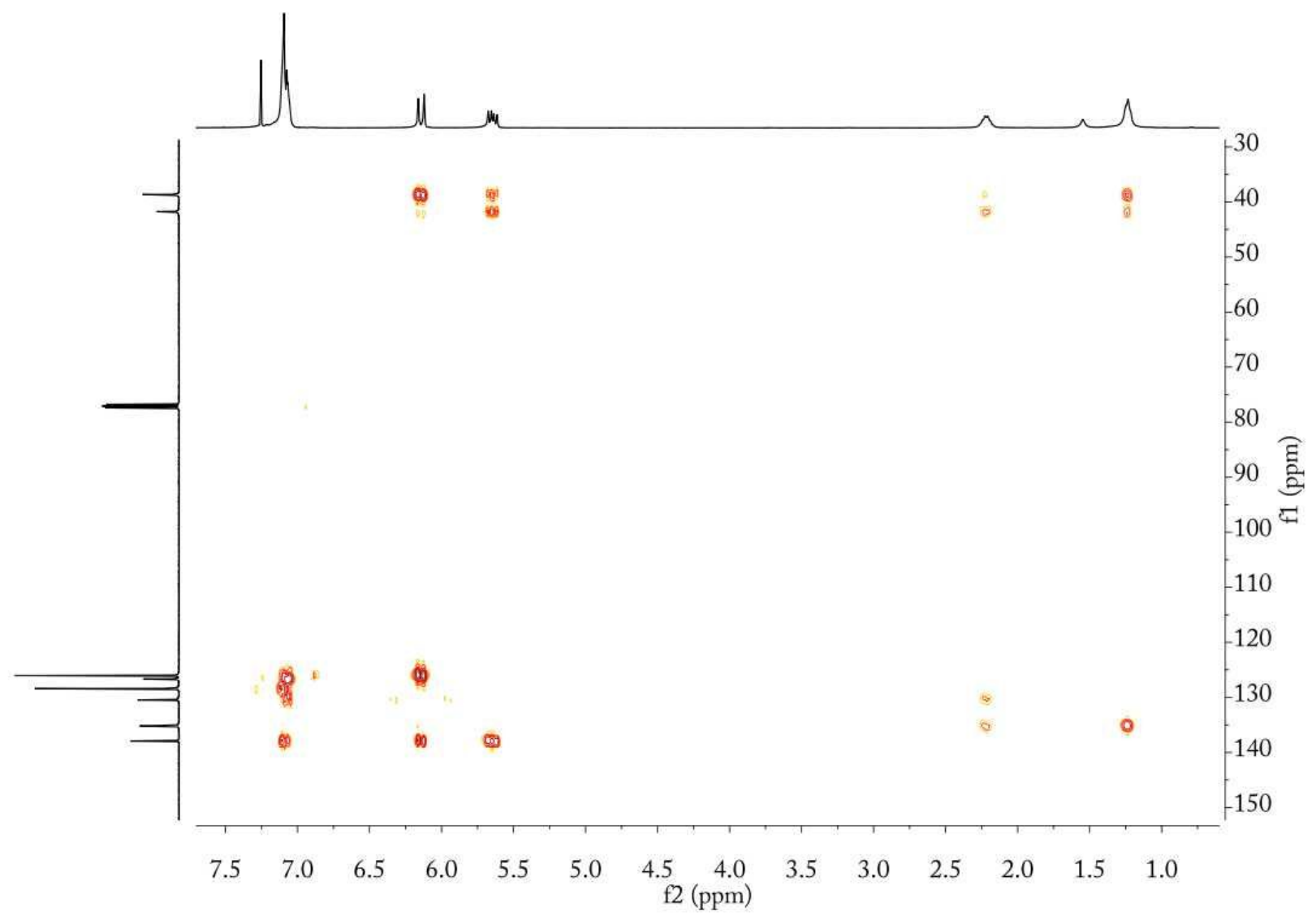

Figure S14 $\mathrm{HMBC}\left(25^{\circ} \mathrm{C}, \mathrm{CDCl}_{3}\right)$ spectrum of 3,4-poly(1PB) (Table $\mathrm{S} 1$, run 1).

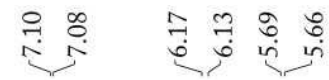

สิ

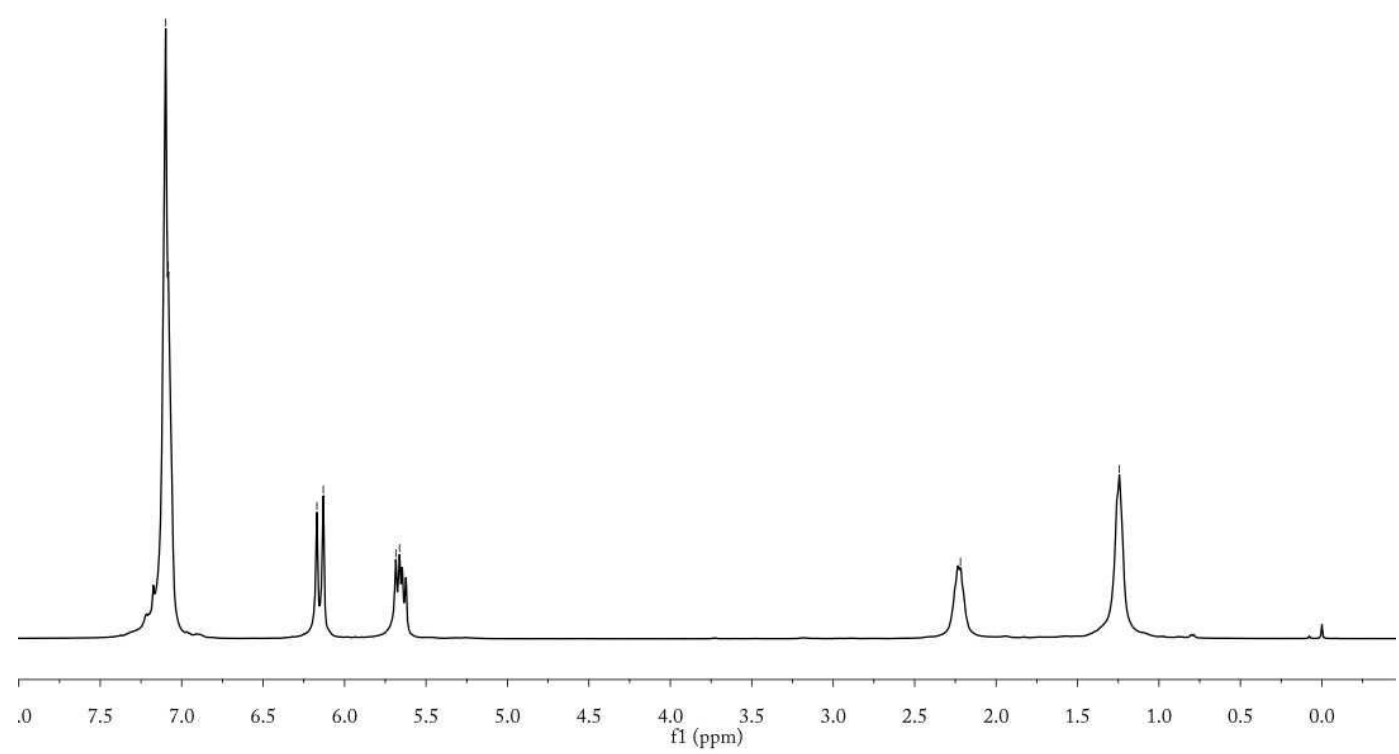

Figure $\mathbf{S 1 5}{ }^{1} \mathrm{H}$ NMR $\left(400 \mathrm{~Hz}, 25{ }^{\circ} \mathrm{C}, \mathrm{CDCl}_{3}\right)$ spectrum of 3,4-poly(1PB) (Table $\mathrm{S} 1$, run 2). 


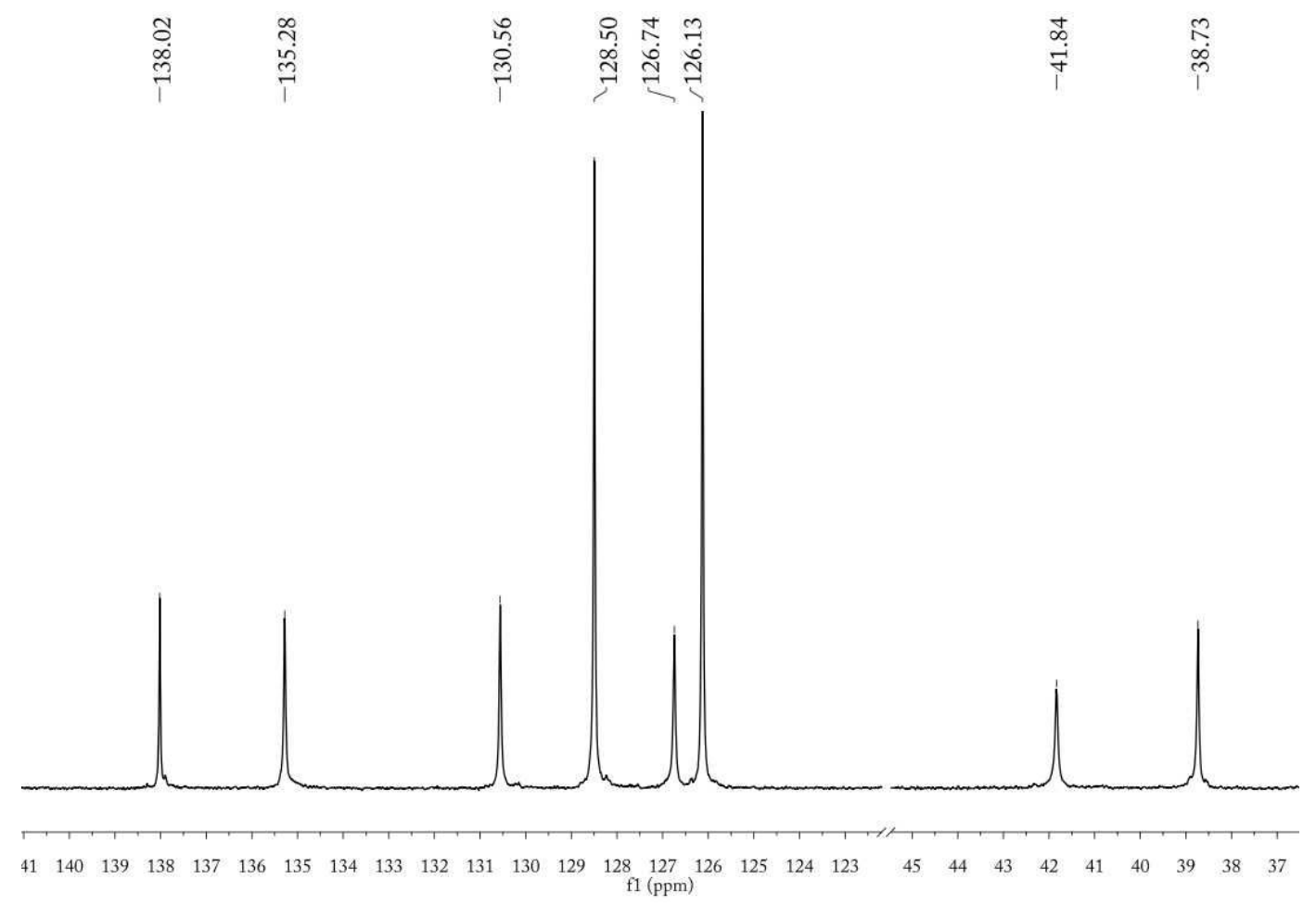

Figure S16 ${ }^{13} \mathrm{C}$ NMR $\left(100 \mathrm{~Hz}, 25{ }^{\circ} \mathrm{C}, \mathrm{CDCl}_{3}\right)$ spectrum of 3,4-poly(1PB) (Table S1, run 2)

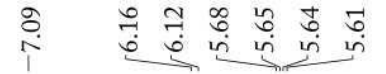

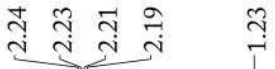

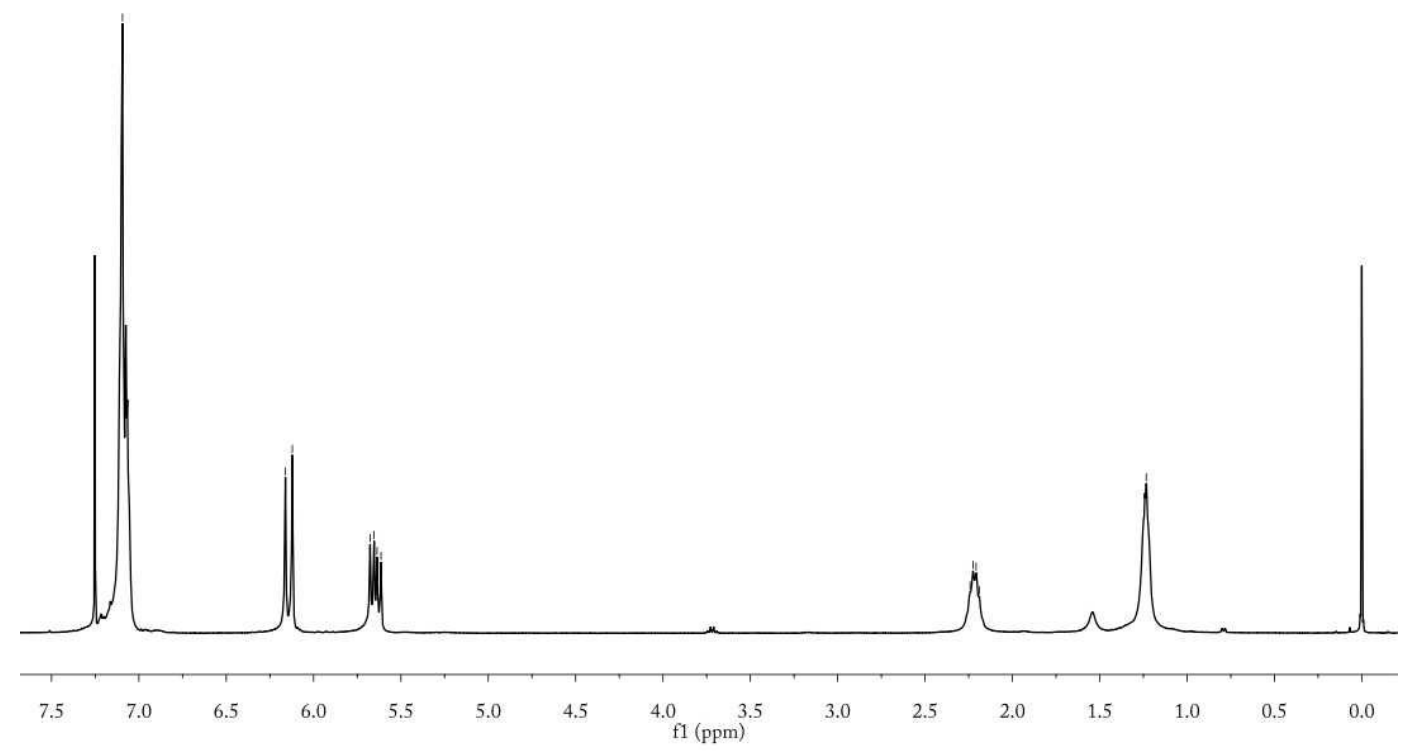

Figure $\mathbf{S 1 7}{ }^{1} \mathrm{H}$ NMR $\left(400 \mathrm{~Hz}, 25{ }^{\circ} \mathrm{C}, \mathrm{CDCl}_{3}\right)$ spectrum of 3,4-poly(1PB) (Table S1, run 3). 


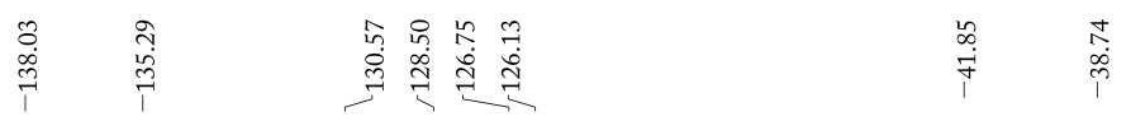

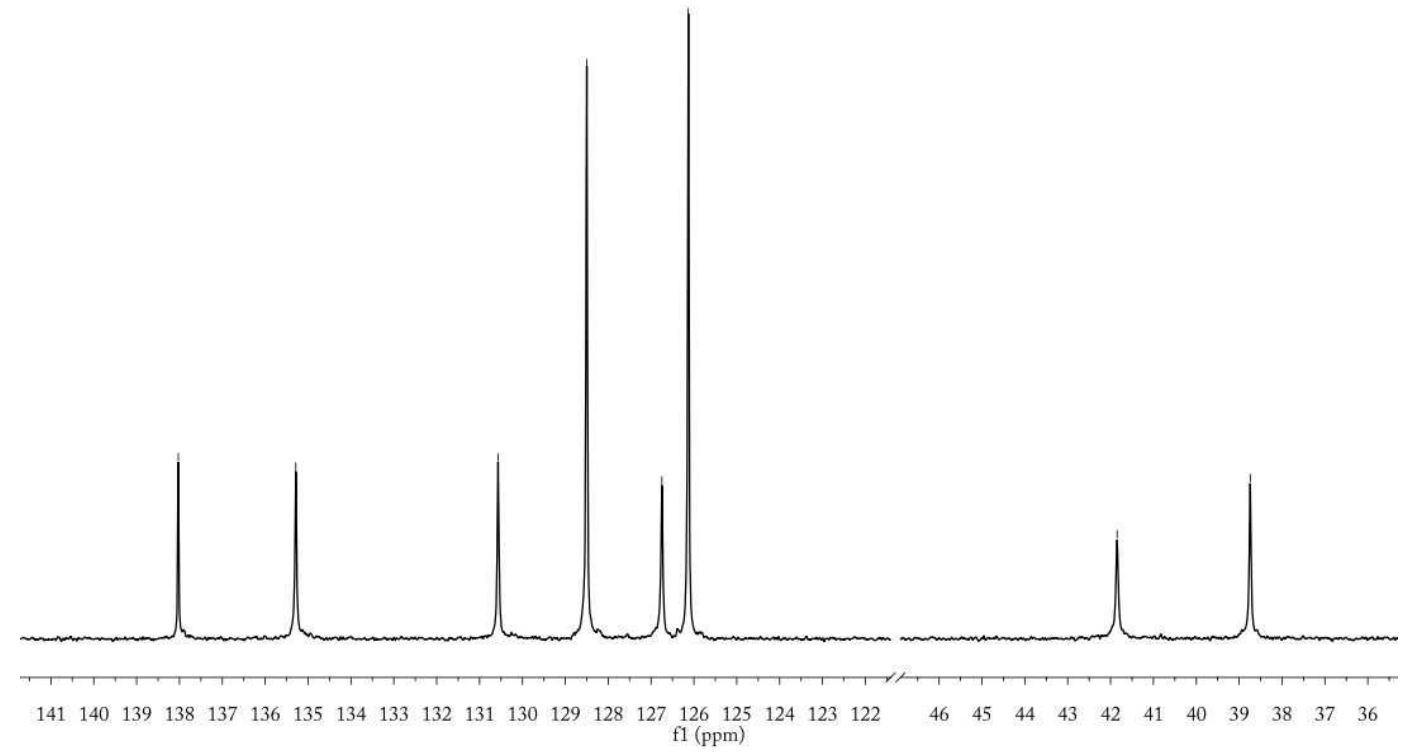

Figure S18 ${ }^{13} \mathrm{C}$ NMR $\left(100 \mathrm{~Hz}, 25^{\circ} \mathrm{C}, \mathrm{CDCl}_{3}\right)$ spectrum of 3,4-poly(1PB) (Table S1, run 3)

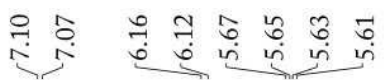

$\stackrel{\text { กิ }}{\text { กิ }}$

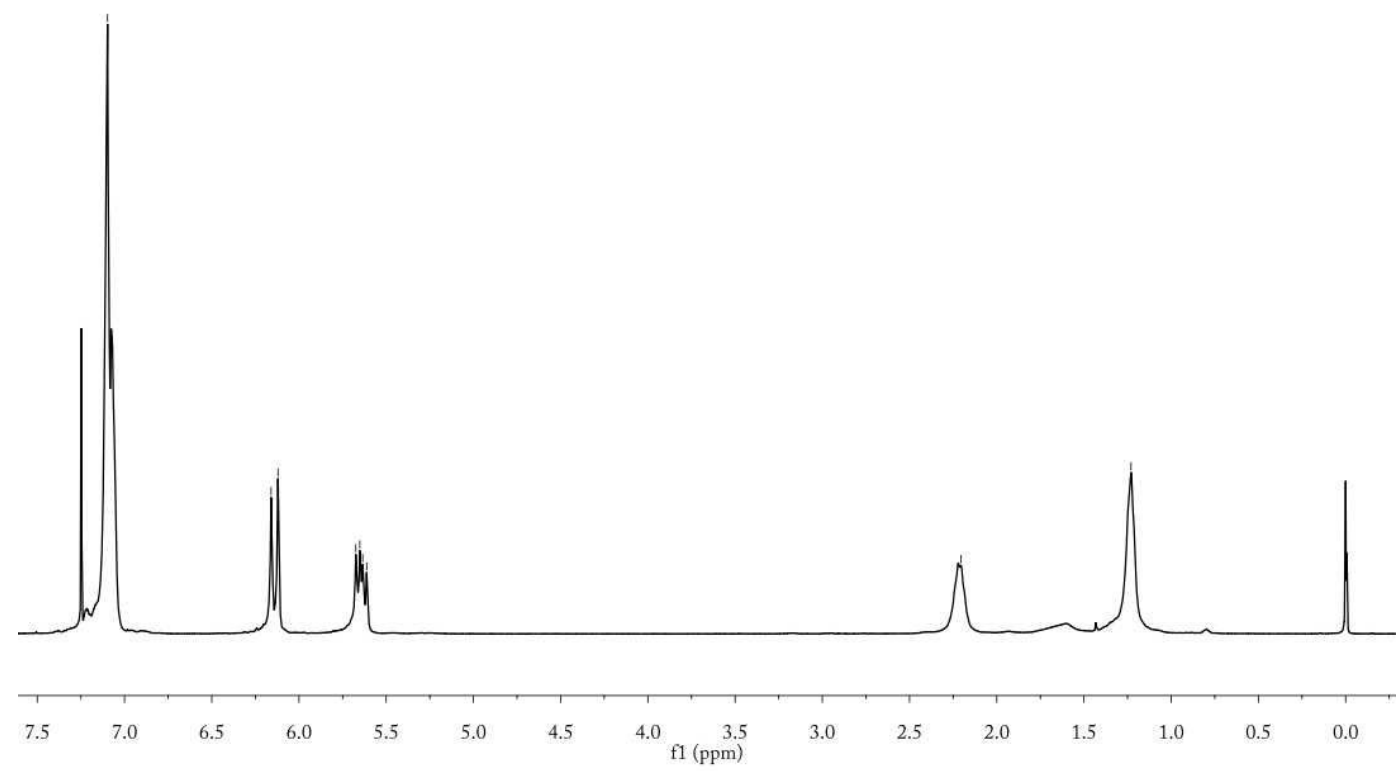

Figure S19 ${ }^{1} \mathrm{H}$ NMR $\left(400 \mathrm{~Hz}, 25^{\circ} \mathrm{C}, \mathrm{CDCl}_{3}\right)$ spectrum of 3,4-poly(1PB) (Table S1, run 4). 


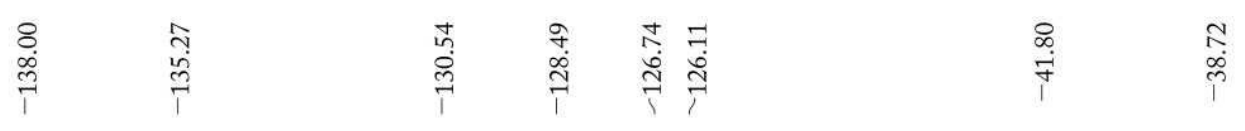

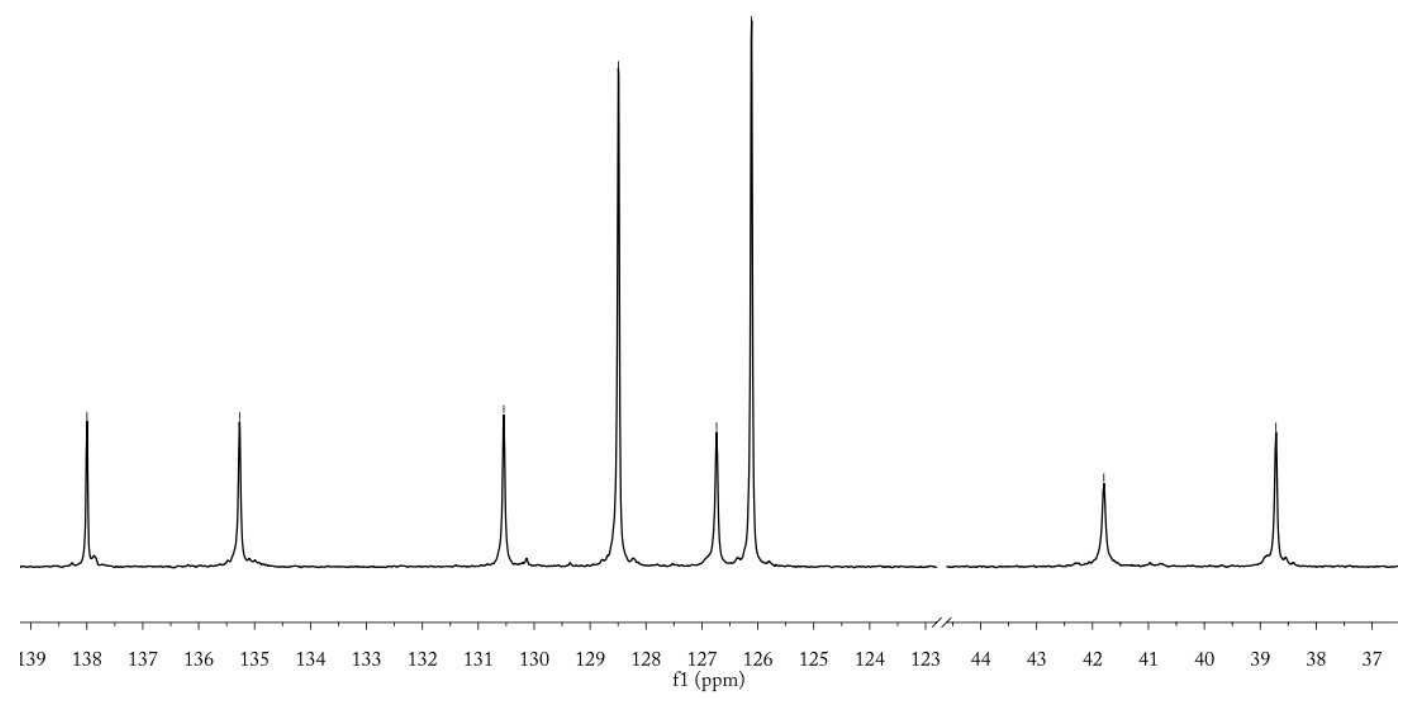

Figure S20 ${ }^{13} \mathrm{C}$ NMR $\left(100 \mathrm{~Hz}, 25^{\circ} \mathrm{C}, \mathrm{CDCl}_{3}\right)$ spectrum of 3,4-poly(1PB) (Table $\mathrm{S} 1$, run 4)

宅

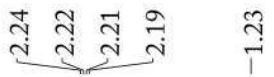

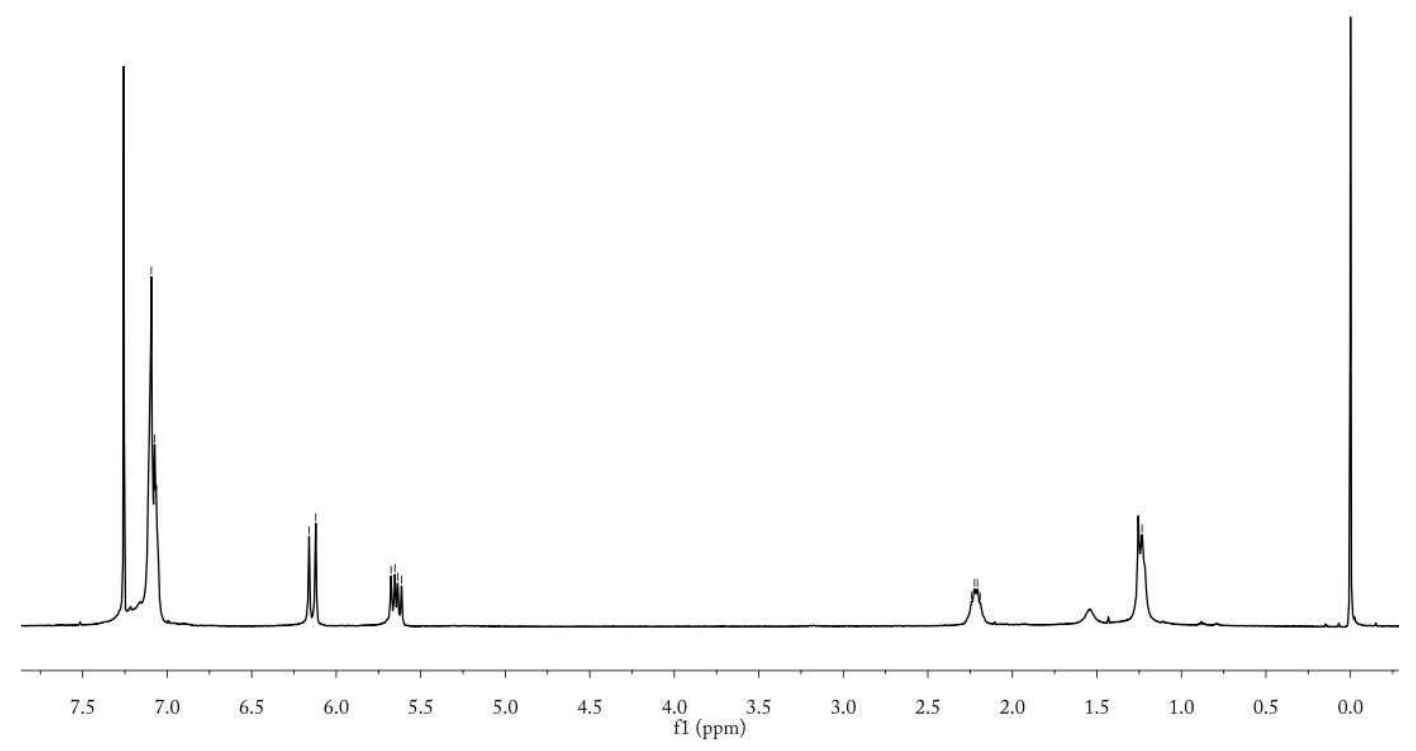

Figure S21 ${ }^{1} \mathrm{H}$ NMR $\left(400 \mathrm{~Hz}, 25^{\circ} \mathrm{C}, \mathrm{CDCl}_{3}\right)$ spectrum of 3,4-poly(1PB) (Table S1, run 5) 


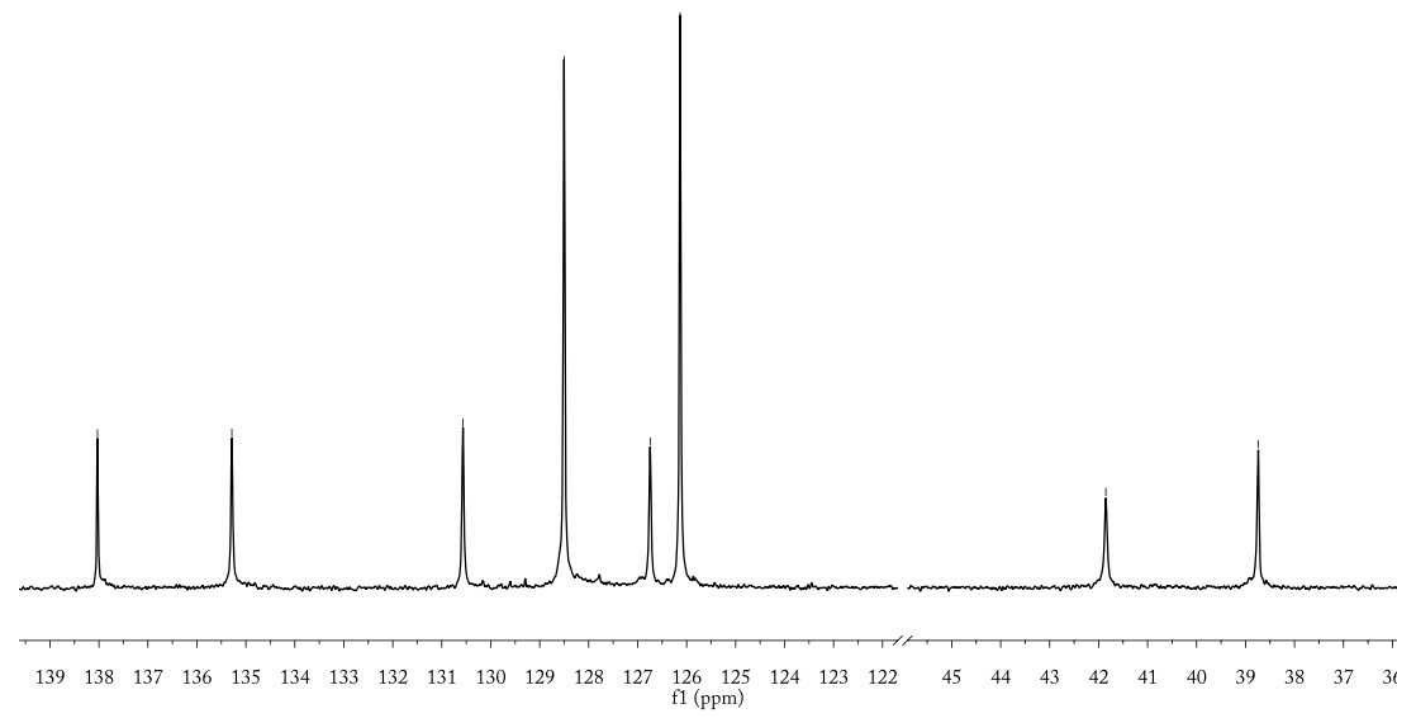

Figure S22 ${ }^{13} \mathrm{C}$ NMR $\left(100 \mathrm{~Hz}, 25{ }^{\circ} \mathrm{C}, \mathrm{CDCl}_{3}\right)$ spectrum of 3,4-poly(1PB) (Table S1, run 5)

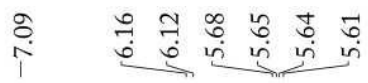

กู่

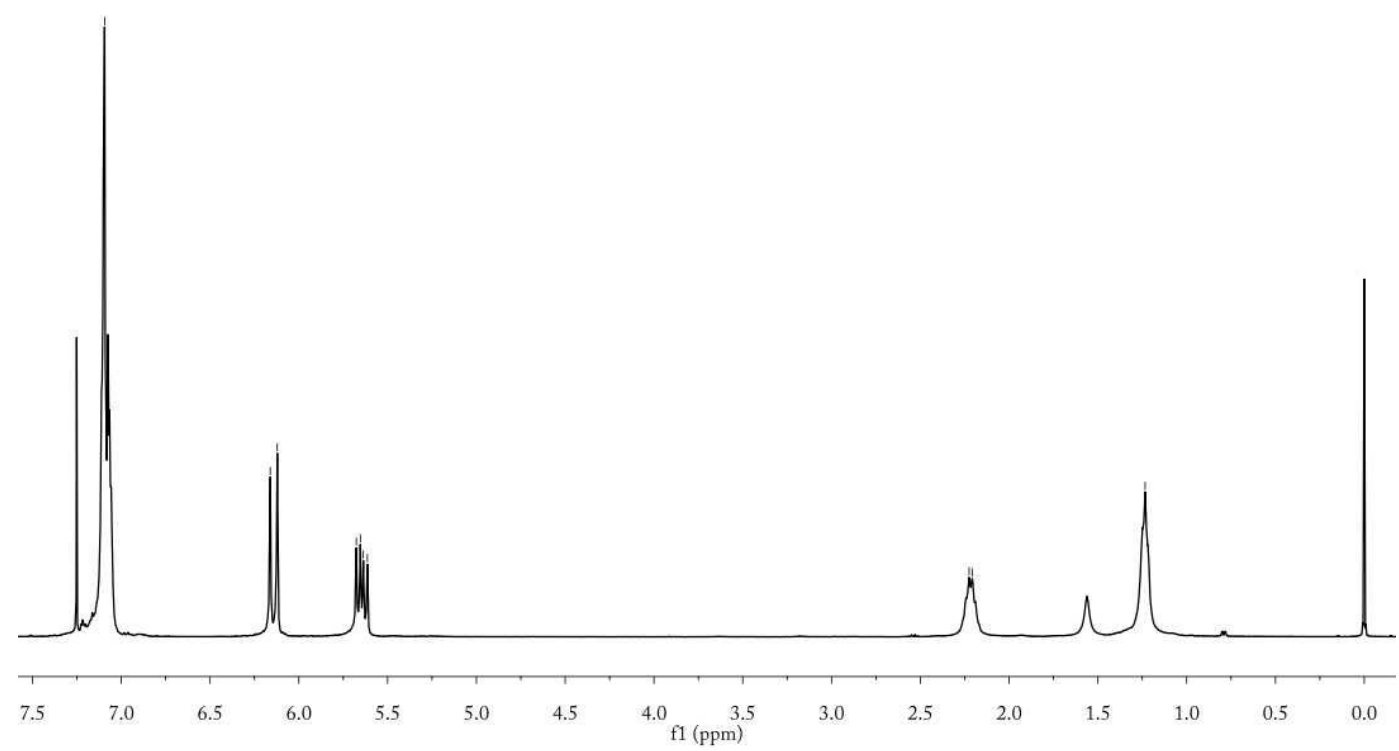

Figure S23 ${ }^{1} \mathrm{H}$ NMR $\left(400 \mathrm{~Hz}, 25^{\circ} \mathrm{C}, \mathrm{CDCl}_{3}\right)$ spectrum of 3,4-poly(1PB) (STable 1, run 6) 


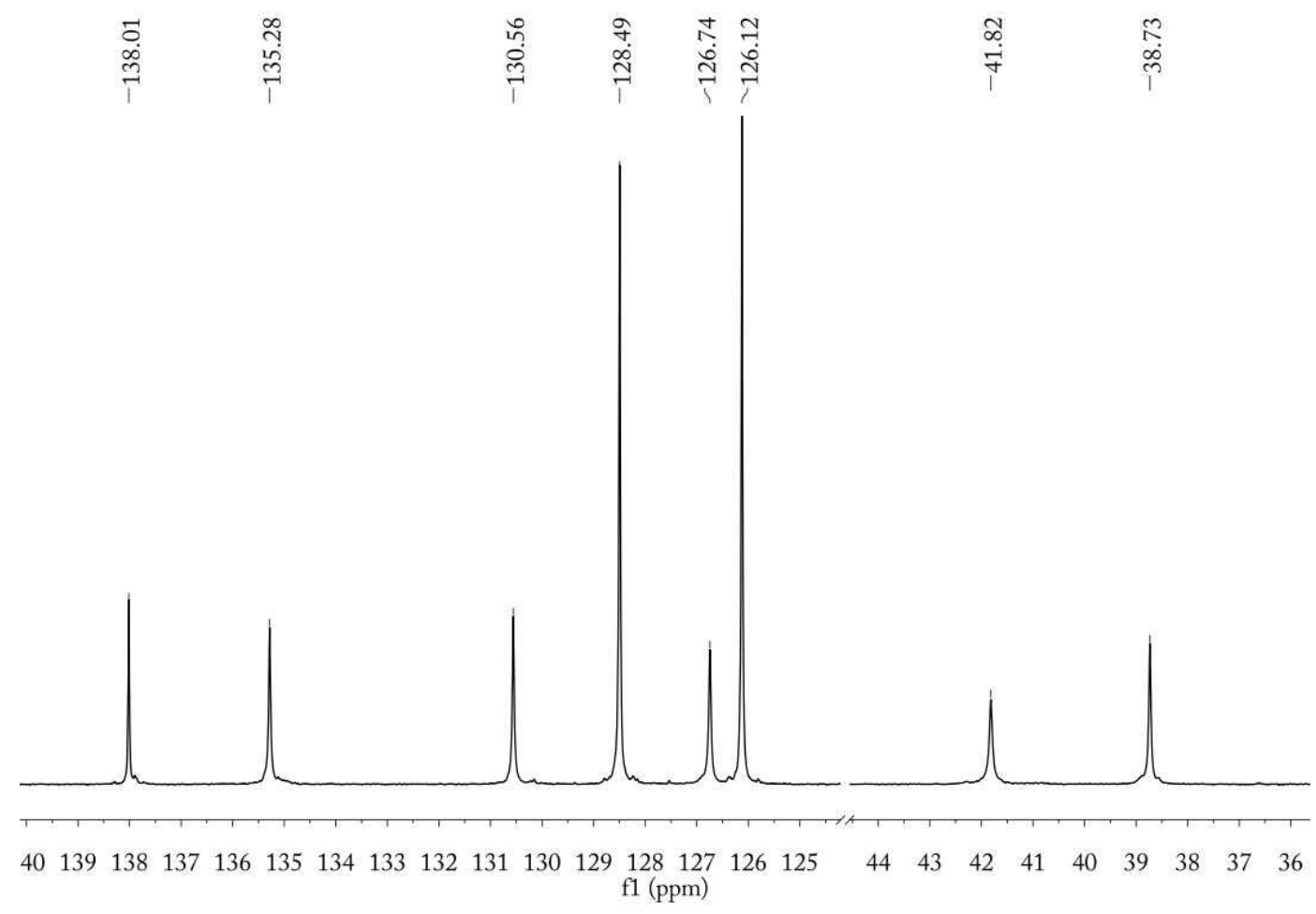

Figure S24 ${ }^{13} \mathrm{C}$ NMR $\left(100 \mathrm{~Hz}, 25{ }^{\circ} \mathrm{C}, \mathrm{CDCl}_{3}\right)$ spectrum of 3,4-poly(1PB) (STable1, run 6)

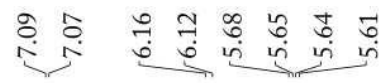

กิ

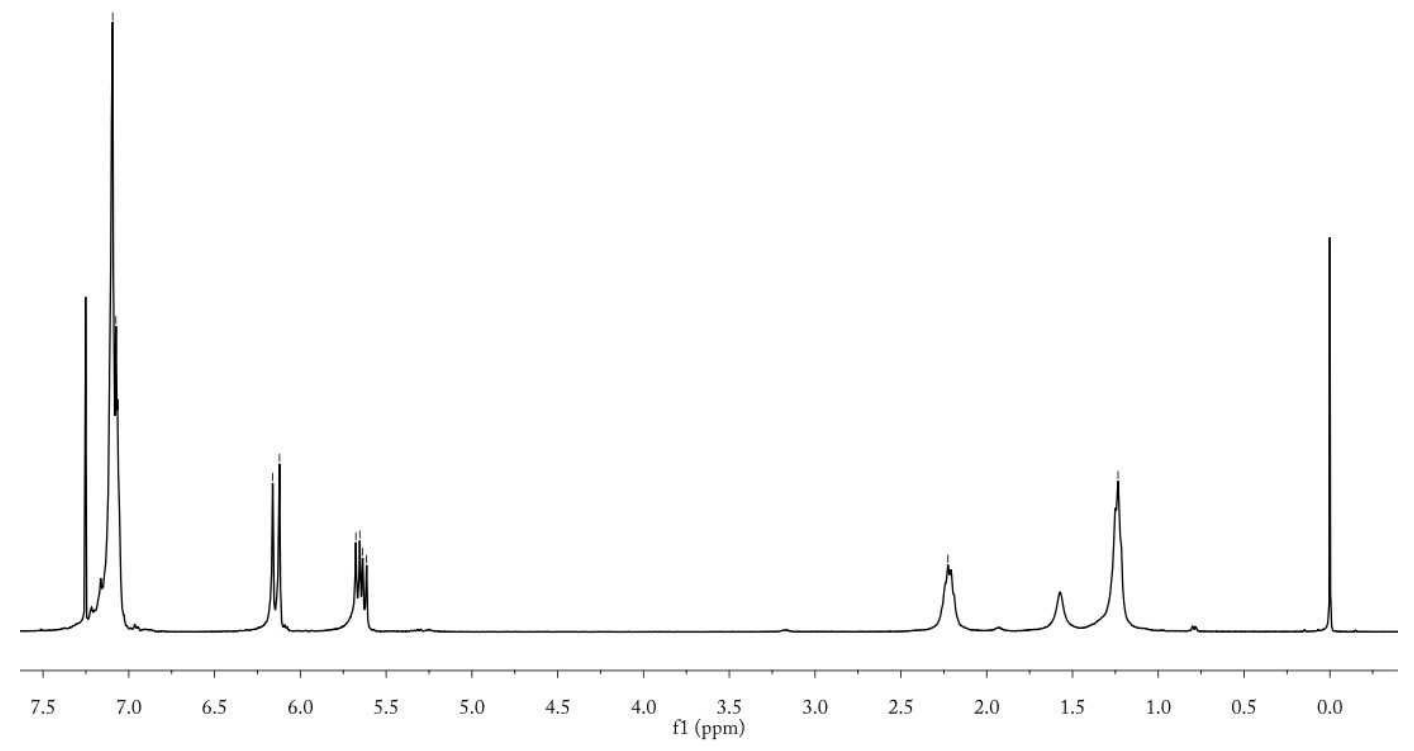

Figure S25 ${ }^{1} \mathrm{H}$ NMR $\left(400 \mathrm{~Hz}, 25{ }^{\circ} \mathrm{C}, \mathrm{CDCl}_{3}\right)$ spectrum of 3,4-poly(1PB) (STable 1, run 7) 


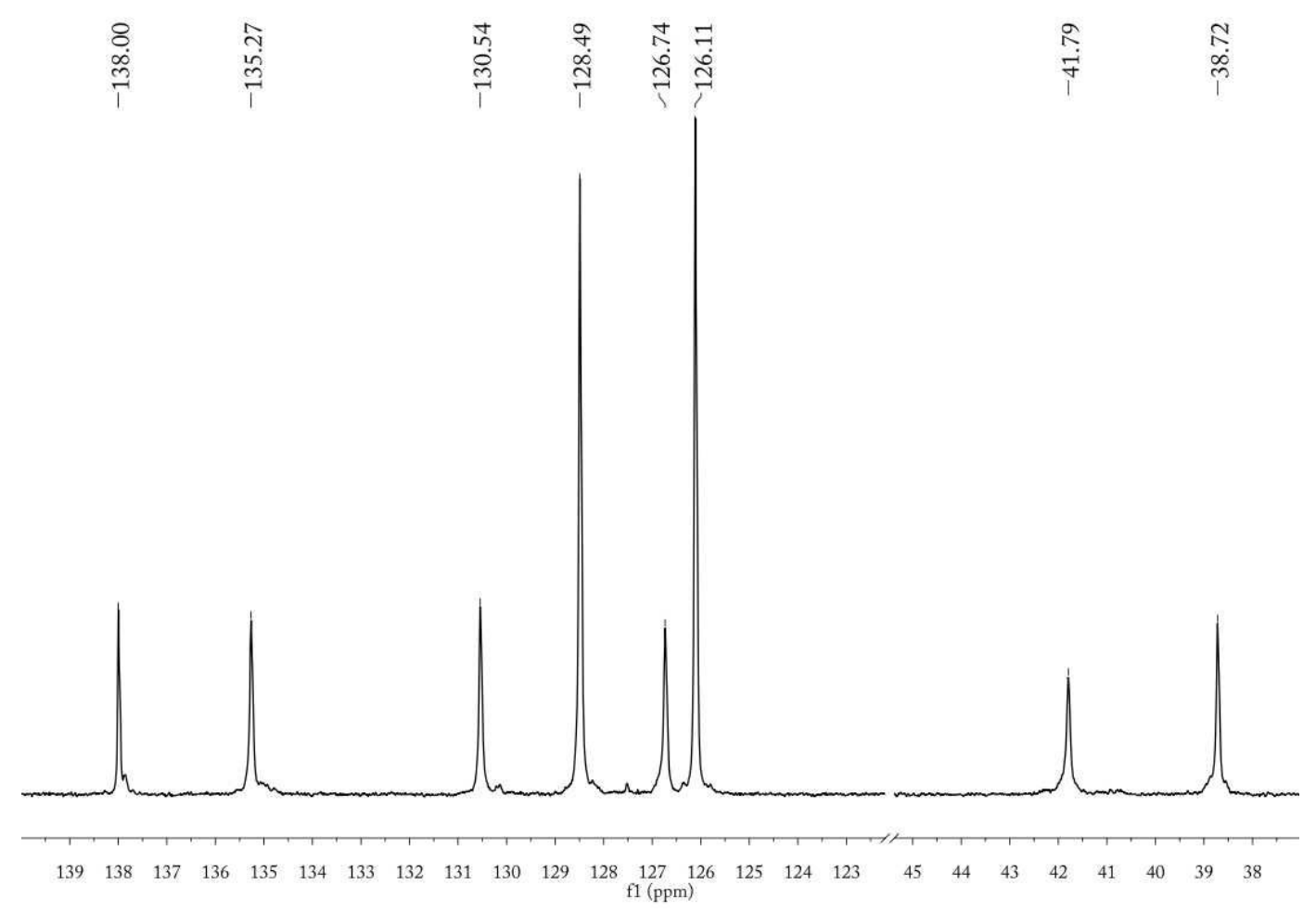

Figure S26 ${ }^{13} \mathrm{C}$ NMR $\left(100 \mathrm{~Hz}, 25{ }^{\circ} \mathrm{C}, \mathrm{CDCl}_{3}\right)$ spectrum of 3, 4-poly(1PB) (STable 1, run 7)

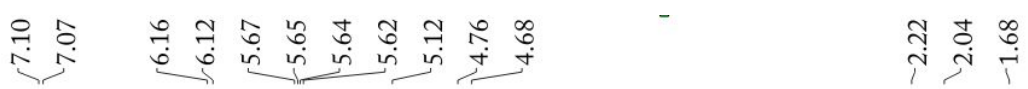

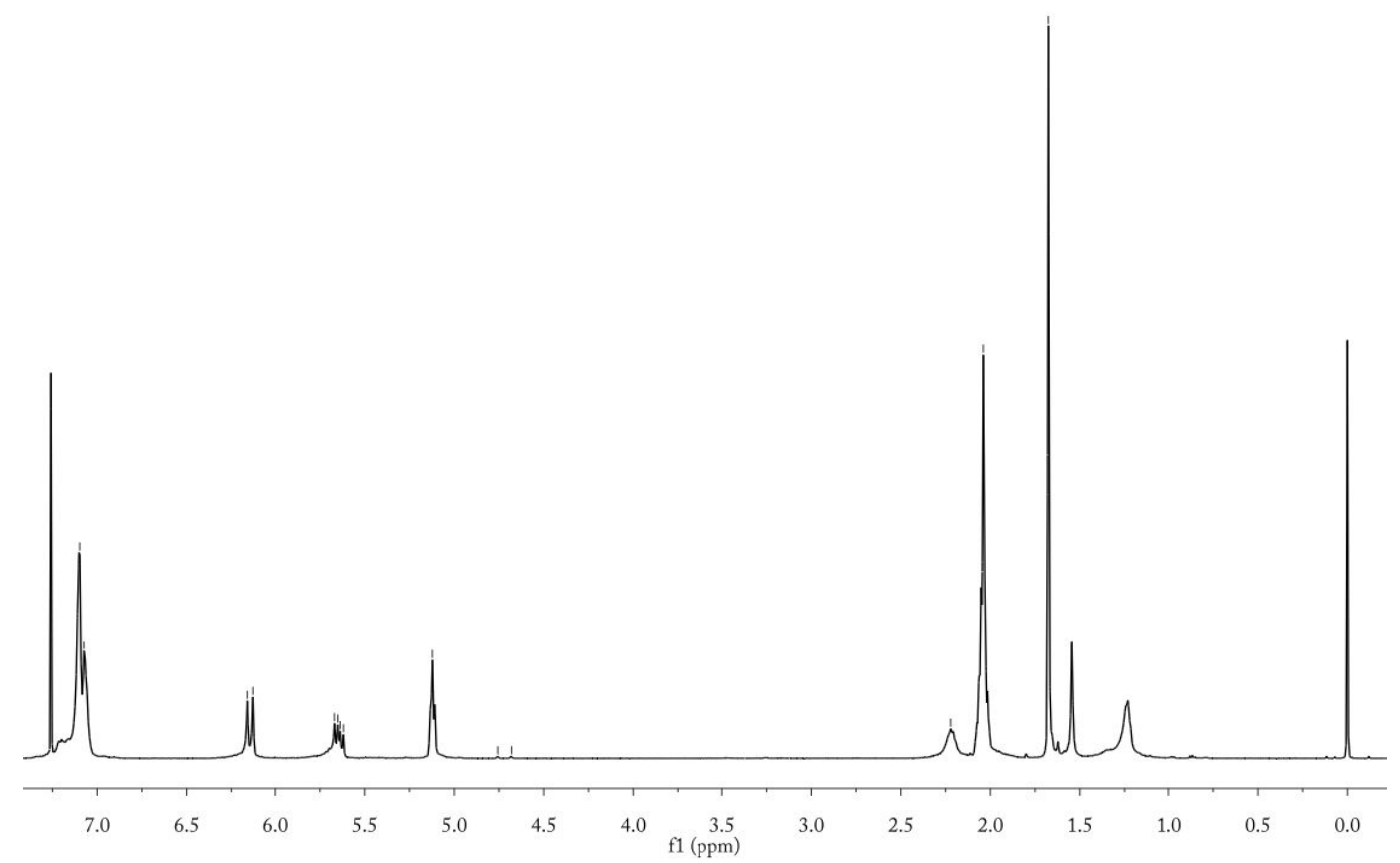

Figure S27 ${ }^{1} \mathrm{H}$ NMR $\left(400 \mathrm{~Hz}, 25^{\circ} \mathrm{C}, \mathrm{CDCl}_{3}\right)$ spectrum of cis-1,4-PIP-block-syndiotactic 3,4-poly(1PB). 


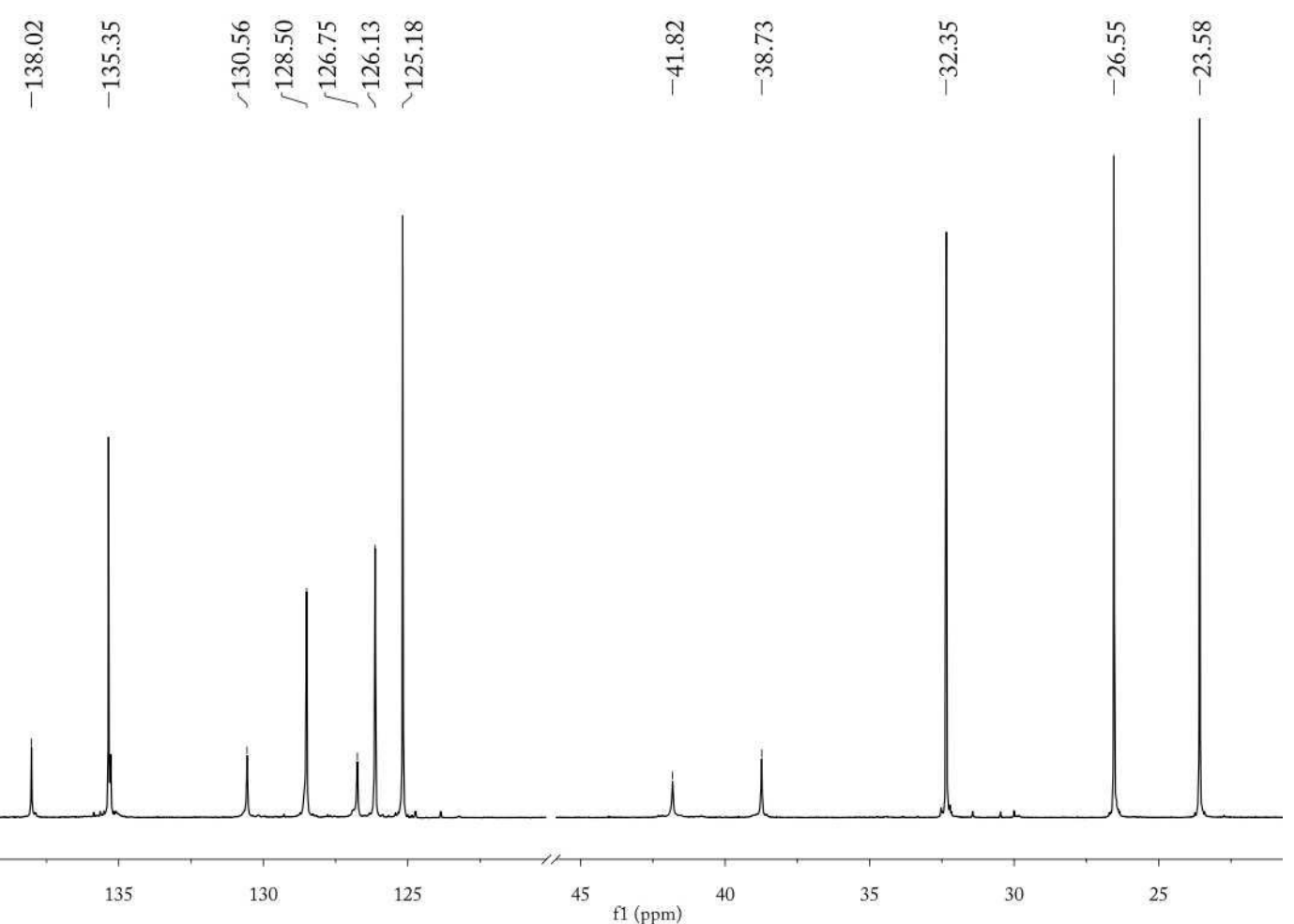

Figure $\mathbf{S 2 8}{ }^{13} \mathrm{C}$ NMR $\left(100 \mathrm{~Hz}, 25{ }^{\circ} \mathrm{C}, \mathrm{CDCl}_{3}\right)$ spectrum of cis-1,4-PIP-block-syndiotactic 3,4-poly(1PB).

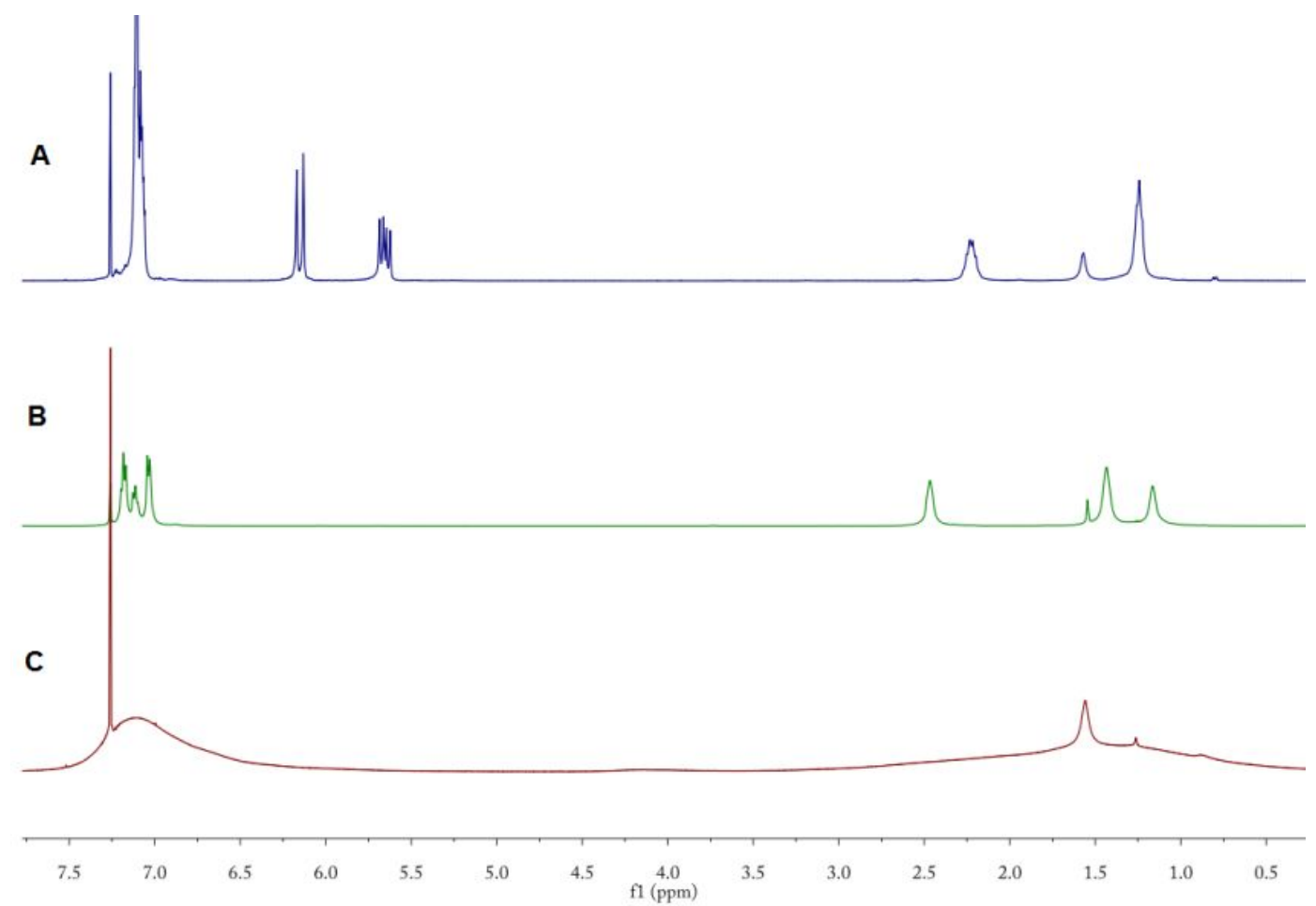

Figure S29 ${ }^{1} \mathrm{H}$ NMR $\left(400 \mathrm{~Hz}, 25^{\circ} \mathrm{C}, \mathrm{CDCl}_{3}\right)$ spectra of syndiotactic 3,4-poly(1PB) (A, Table S1, run 2), hydrogenated syndiotactic 3,4-poly(1PB) (B) and cyclized syndiotactic 3,4-poly(1PB) (C). 


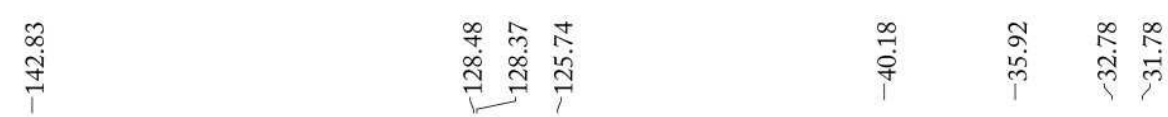

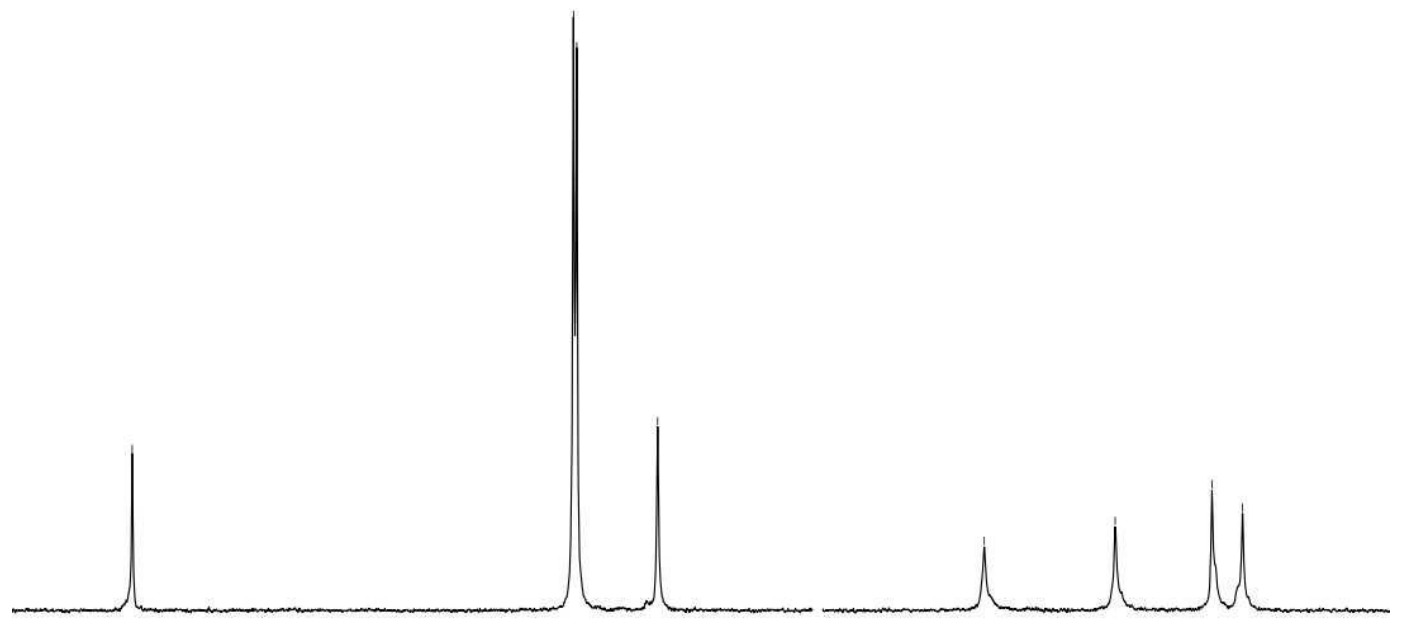

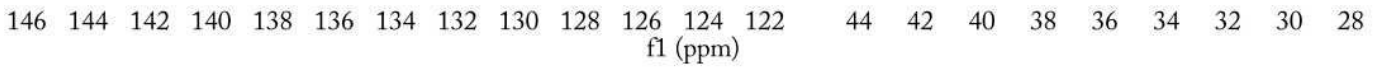

Figure S30 ${ }^{13} \mathrm{C}$ NMR $\left(100 \mathrm{~Hz}, 25{ }^{\circ} \mathrm{C}, \mathrm{CDCl}_{3}\right)$ spectrum of hydrogenated syndiotactic 3,4-poly(1PB).
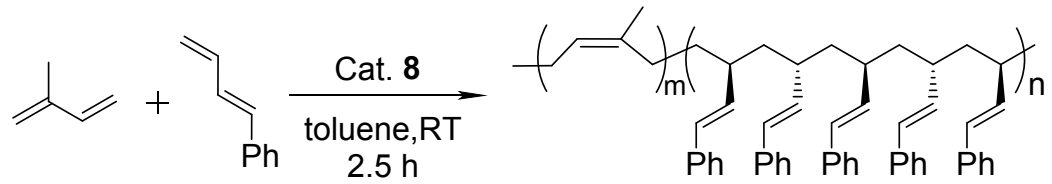

98.9\% cis-1,4-PIP (51 mol\%)

$>99.0 \%$ 3,4-Poly(1PB), $94.4 \%$ rrrr

$M_{\mathrm{n}}=8.74 \times 10^{4}, M_{\mathrm{w}} / M_{n}=1.58$

Scheme S1. Regio- and stereo-selective copolymerization of 1PB and IP with precursor 8. 


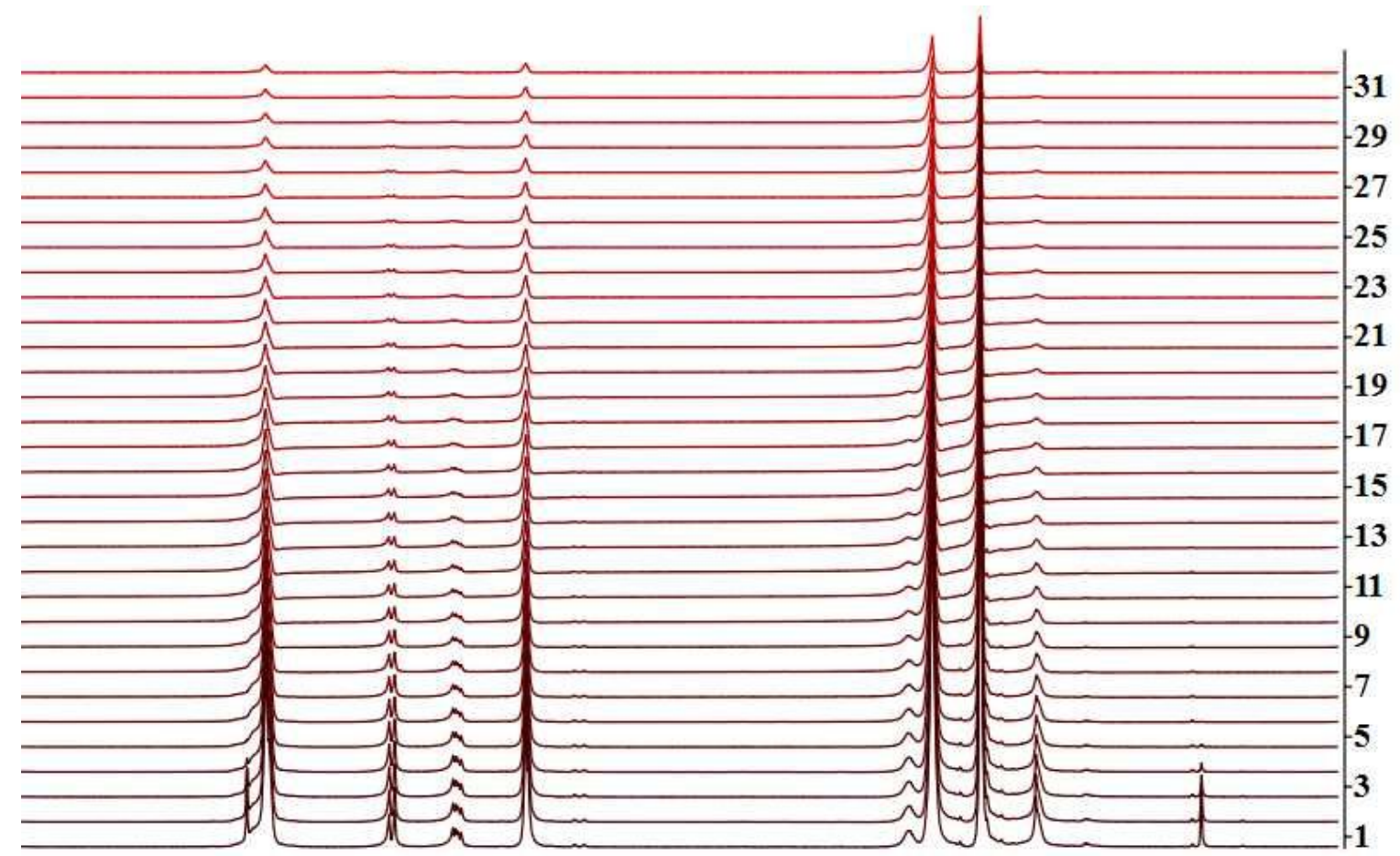

\section{$\begin{array}{lllllllllllllllllll}8.5 & 8.0 & 7.5 & 7.0 & 6.5 & 6.0 & 5.5 & 5.0 & 4.5 & 4.0 & 3.5 & 3.0 & 2.5 & 2.0 & 1.5 & 1.0 & 0.5 & 0.0 & -0.5\end{array}$ chemical shift (ppm)}

Figure S31 DOSY spectrum of cis-1,4-PIP-block-syndiotactic 3,4-poly(1PB) in $\mathrm{CDCl}_{3}$ at $25^{\circ} \mathrm{C}$ (relaxation time $=1 \mathrm{~s}$, diffusion time $=0.2 \mathrm{~s}$ )
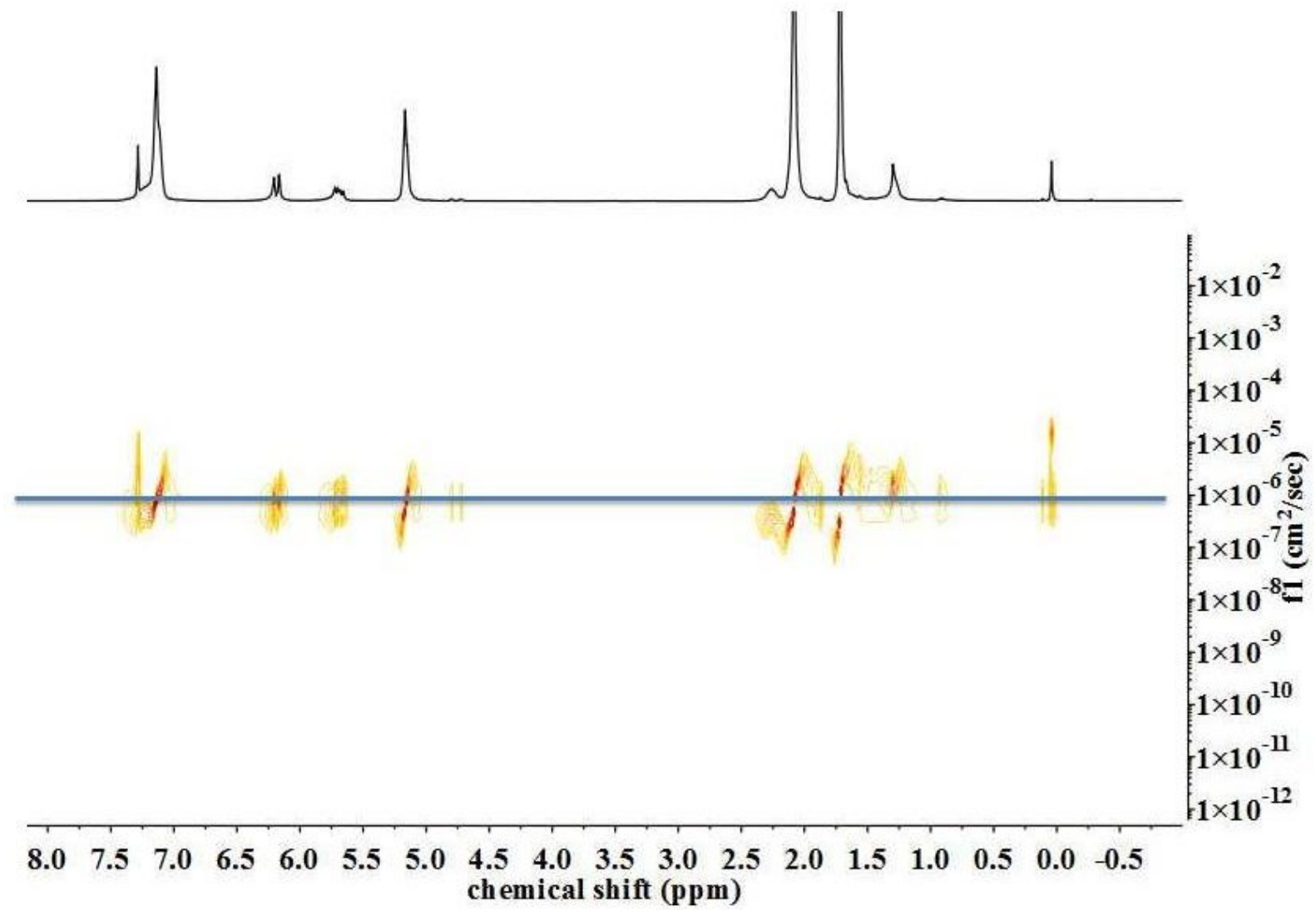

Figure S32 Diffusion coefficient from DOSY spectrum of of cis-1,4-PIP-block-syndiotactic 3,4-poly(1PB) in $\mathrm{CDCl}_{3}$ at $25^{\circ} \mathrm{C}$ (relaxation time $=1 \mathrm{~s}$, diffusion time $=0.2 \mathrm{~s}$ diffusion units: $\mathrm{cm}^{2} \mathrm{~s}^{-1}$ ). 


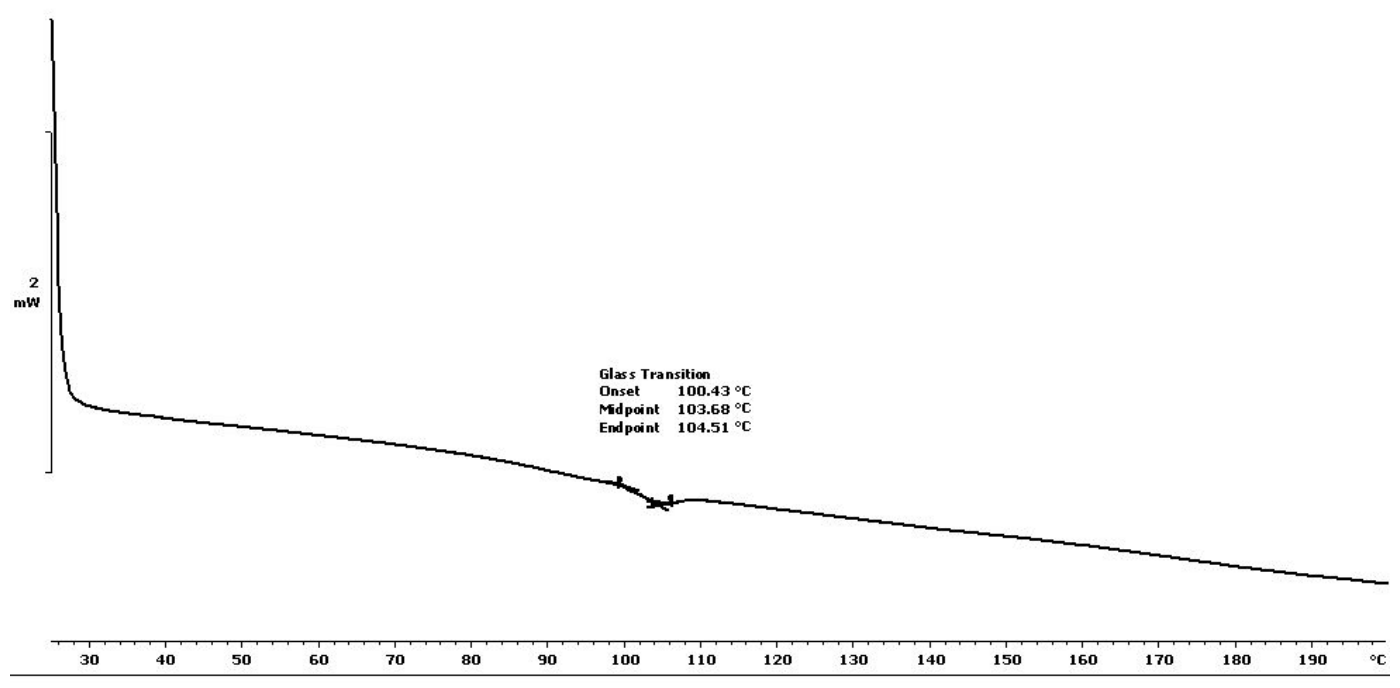

Figure S33 DSC curve of syndiotactic 3,4-poly(1PB) (STable 1, run 2)

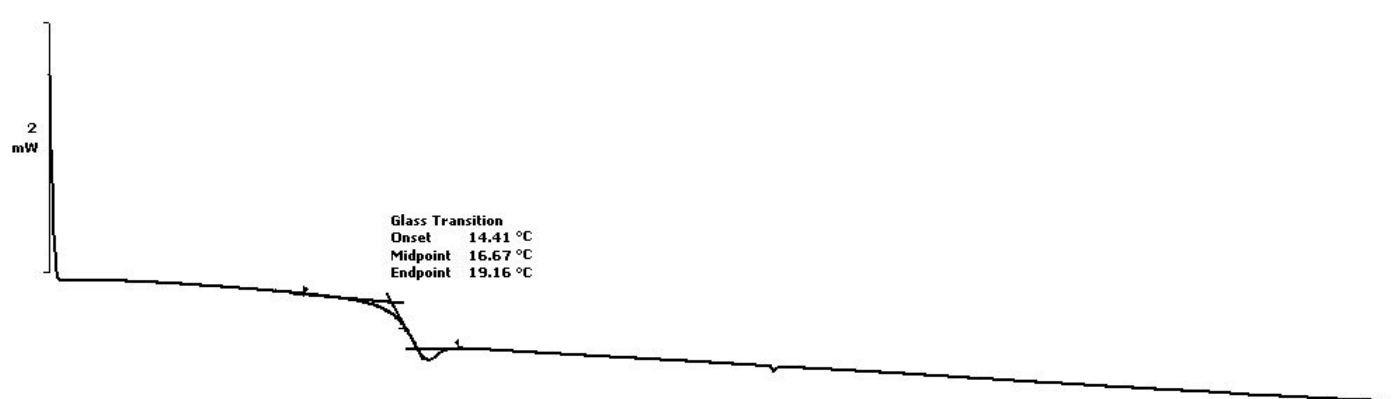

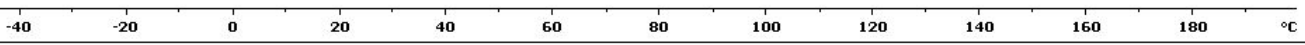

Figure S34 DSC curve of hydrogenated syndiotactic 3,4-poly(1PB)

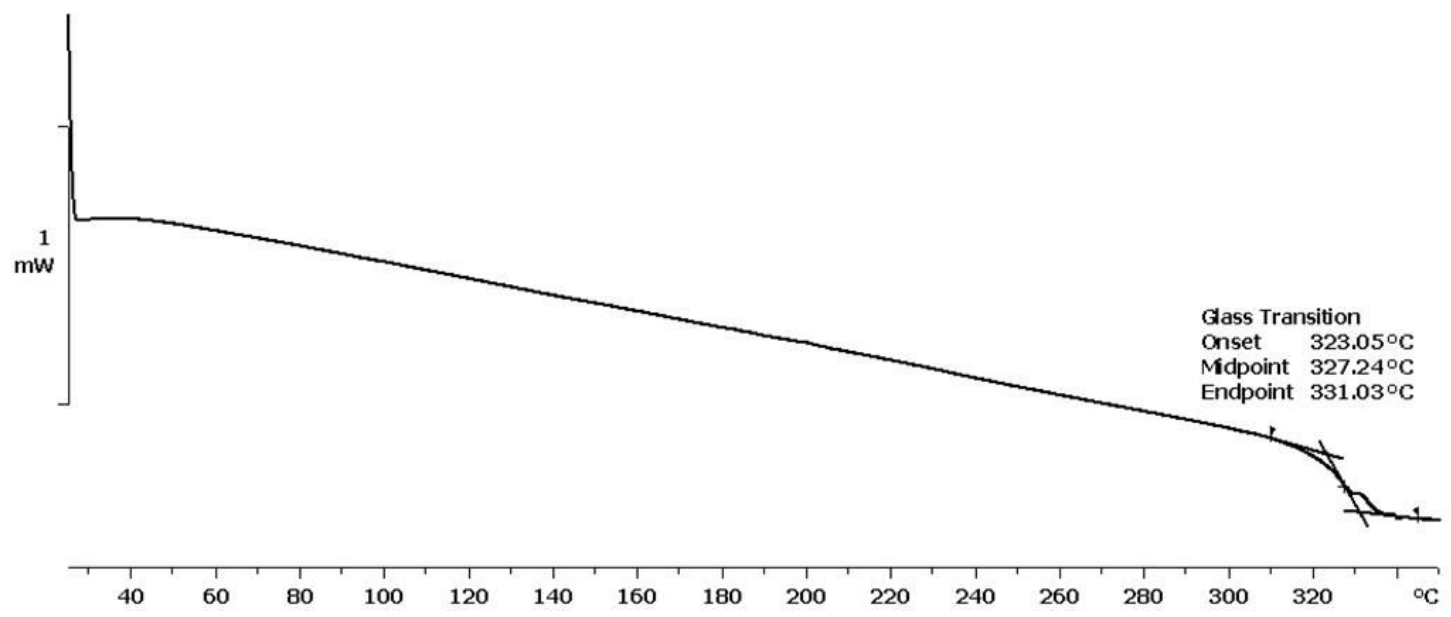

Figure S35 DSC curve of cyclized syndiotactic 3,4-poly(1PB). 


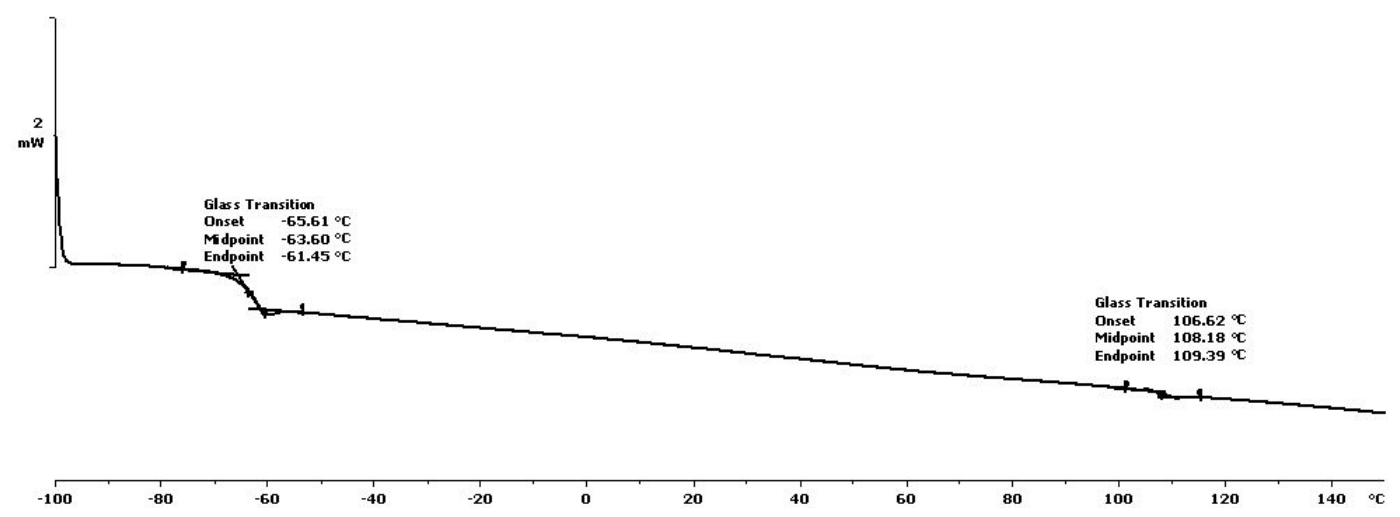

Figure S36 DSC curve of cis-1,4-PIP-block-syndiotactic 3,4-poly(1PB).

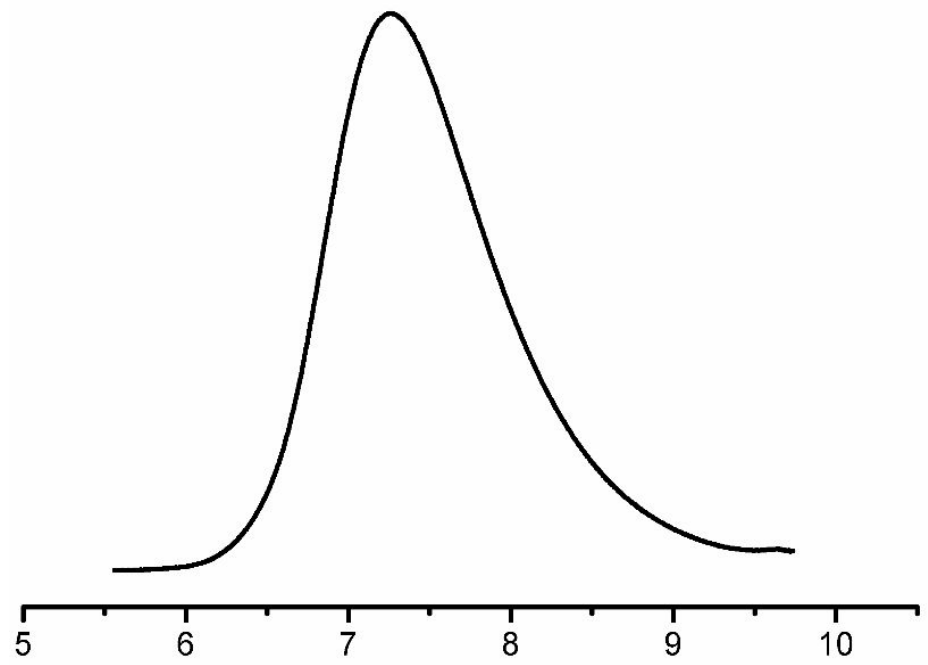

Figure S37 GPC curve of cis-1,4-PIP-block-syndiotactic 3,4-poly(1PB). 


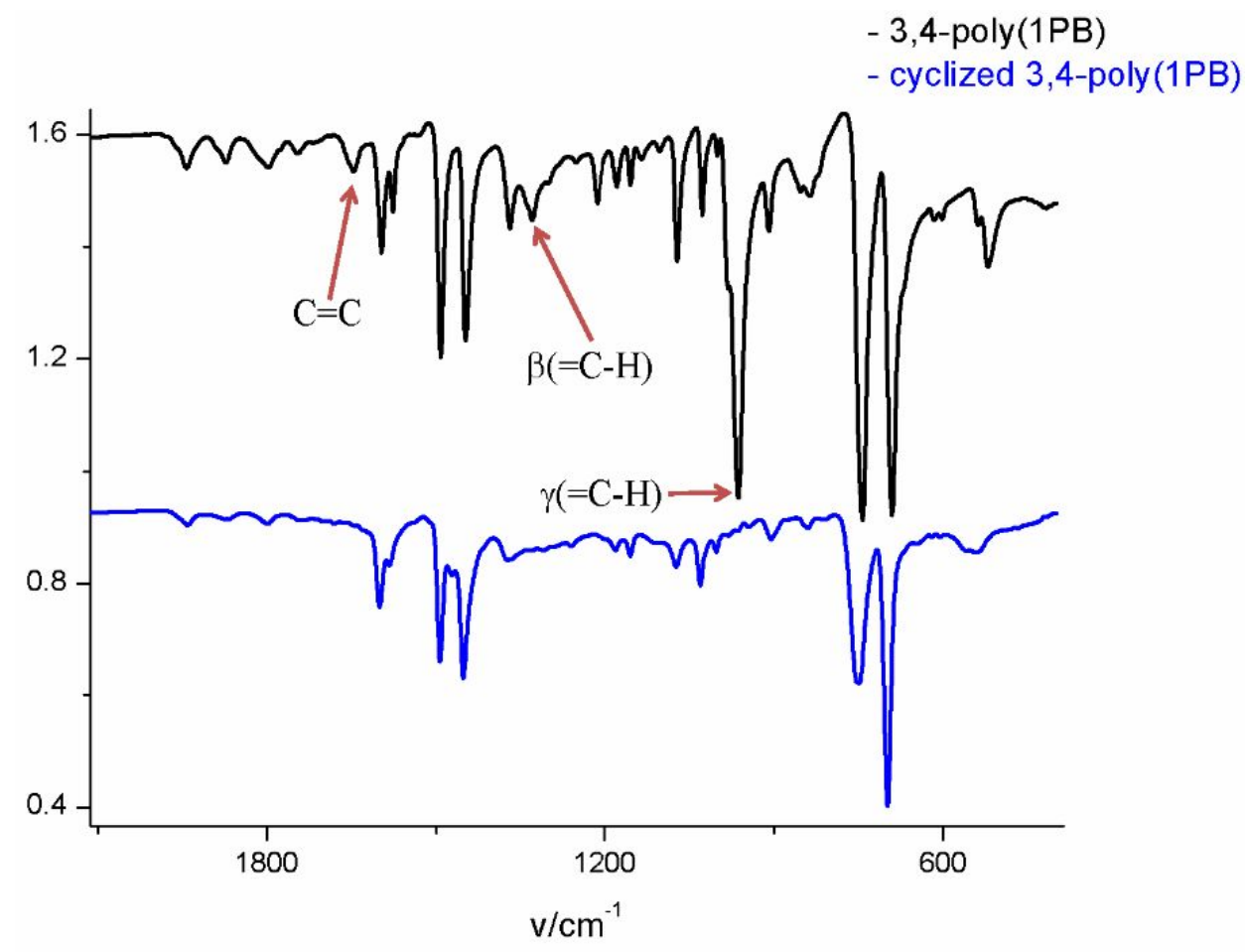

Figure S38 FTIR stack plots of cyclized (below) and uncyclized syndiotactic 3,4-(1PB) (up). 

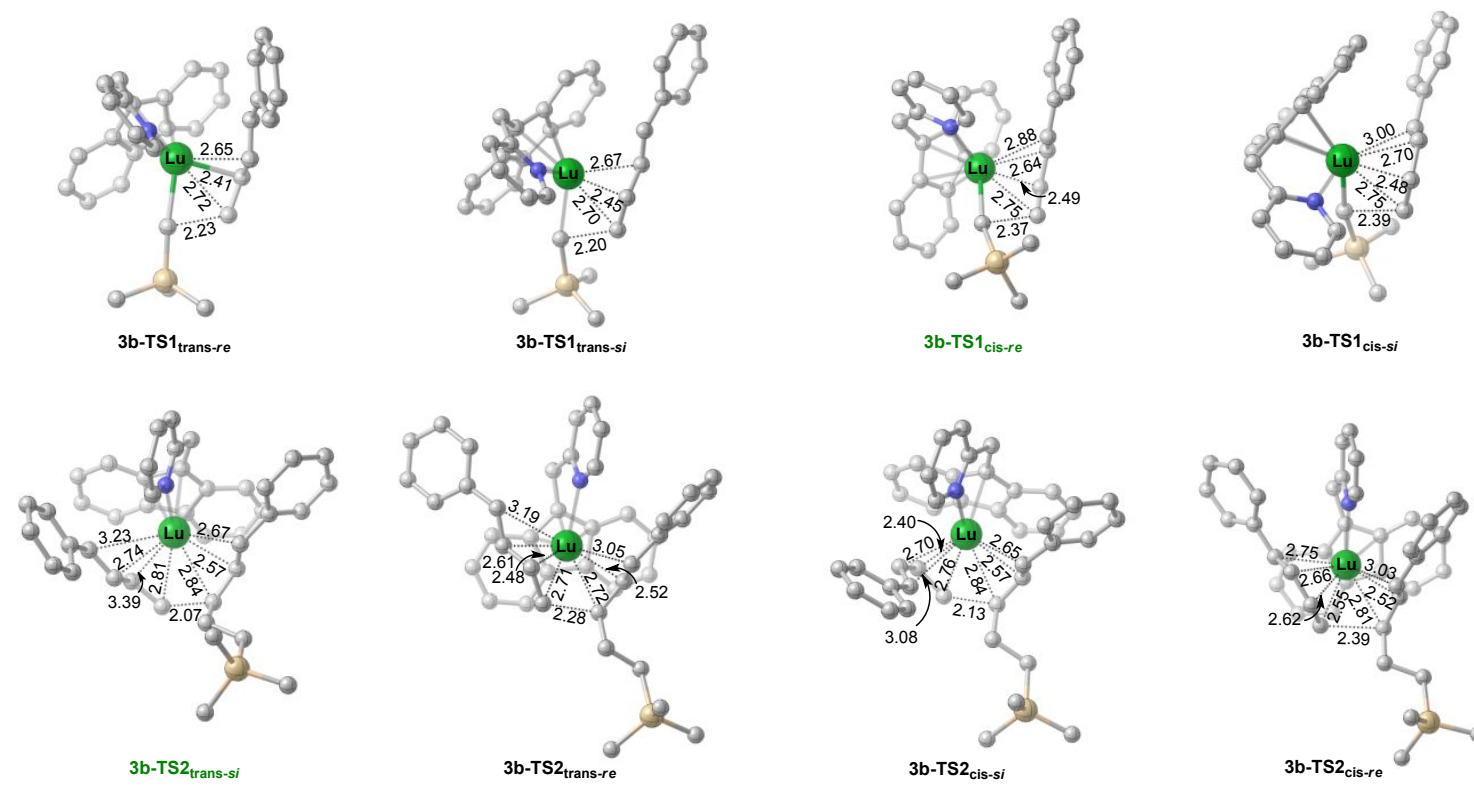

3b-TS1 $1_{\text {cis-re }}$
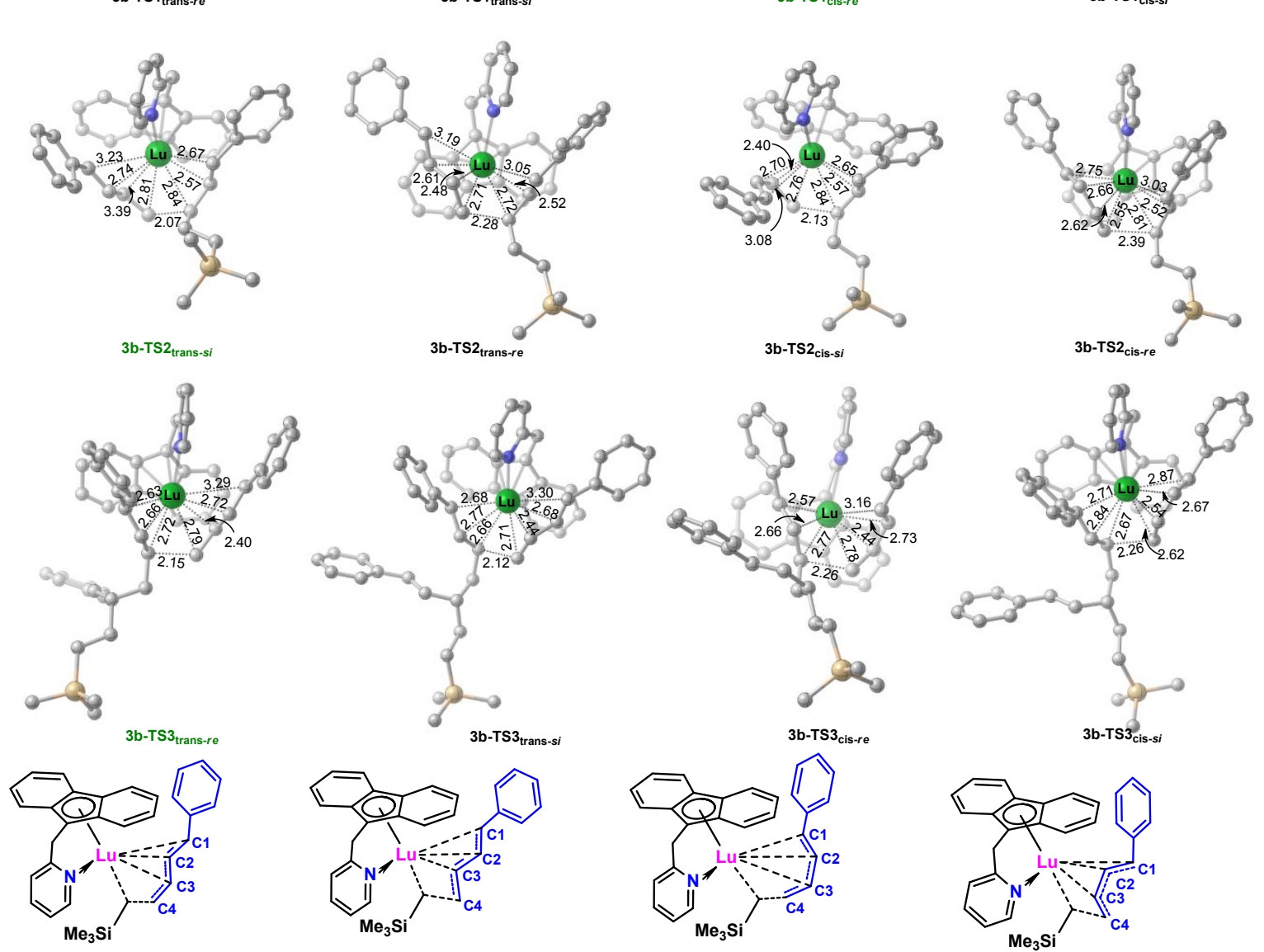

3b-TS1 $1_{\text {trans-re }}$

$(6.5 \mathrm{kcal} / \mathrm{mol})$

3b-TS $1_{\text {trans-si }}$

$(11.3 \mathrm{kcal} / \mathrm{mol})$
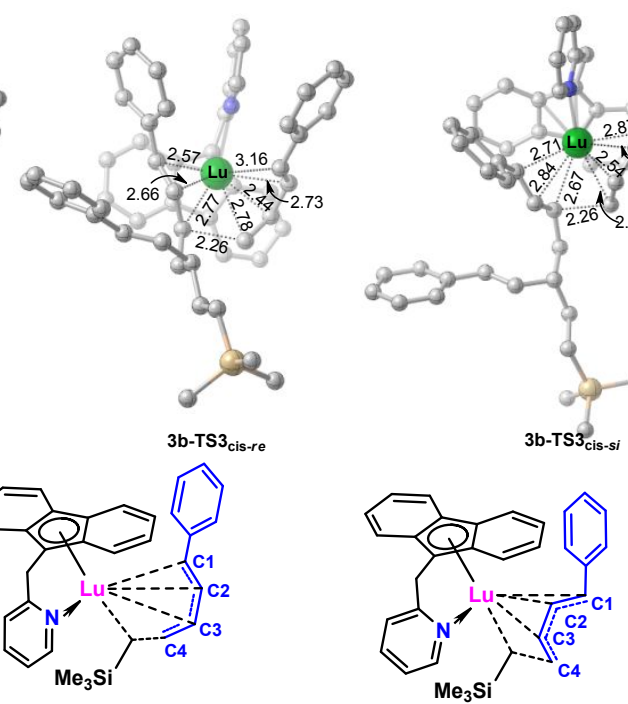

3b-TS $1_{\text {cis-re }}$

(5.7 kcal/mol)

3b-TS1 $1_{\text {cis-si }}$
$(7.8 \mathrm{kcal} / \mathrm{mol})$

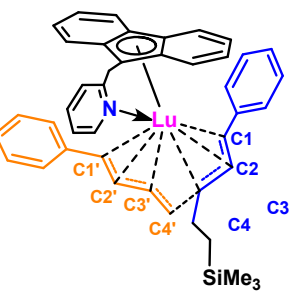

3b-TS2 $2_{\text {trans-si }}$

(-9.8 kcal/mol)

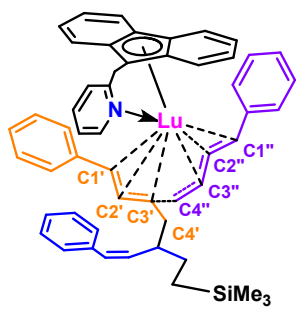

$3 \mathrm{~b}-\mathrm{TS} 3_{\text {trans-re }}$

(-24.4 kcal $/ \mathrm{mol})$

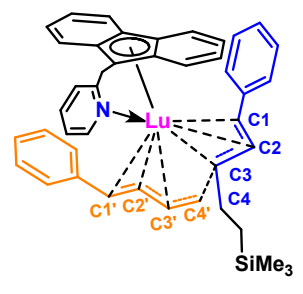

3b-TS2 $2_{\text {trans-re }}$

$(-5.9 \mathrm{kcal} / \mathrm{mol})$

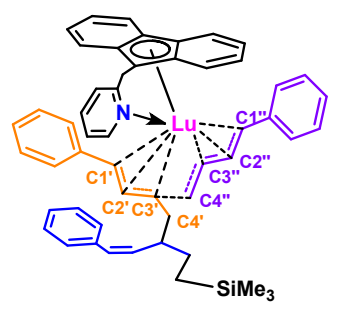

3b-TS3 $3_{\text {trans-si }}$

$(-12.7 \mathrm{kcal} / \mathrm{mol})$

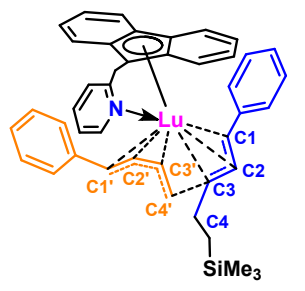

3b-TS2 ${ }_{\text {cis-si }}$

$(-10.2 \mathrm{kcal} / \mathrm{mol})$

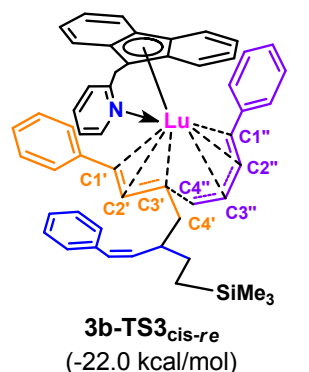

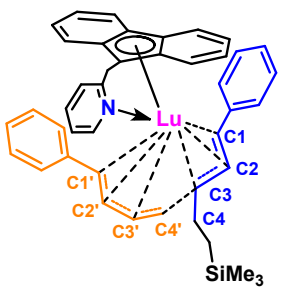

3b-TS2 $2_{\text {cis-re }}$

$(-8.5 \mathrm{kcal} / \mathrm{mol})$

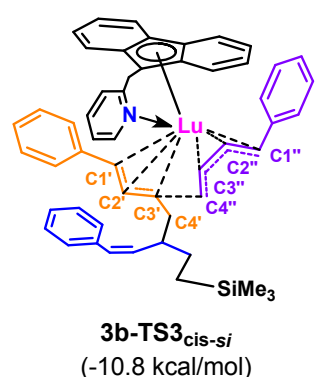

Figure S39 Optimized structures of transition states involved in Figure 1. Energies are relative to corresponding cationic species cat3b and $1 \mathrm{~PB}$. All hydrogen atoms are omitted for clarity. 


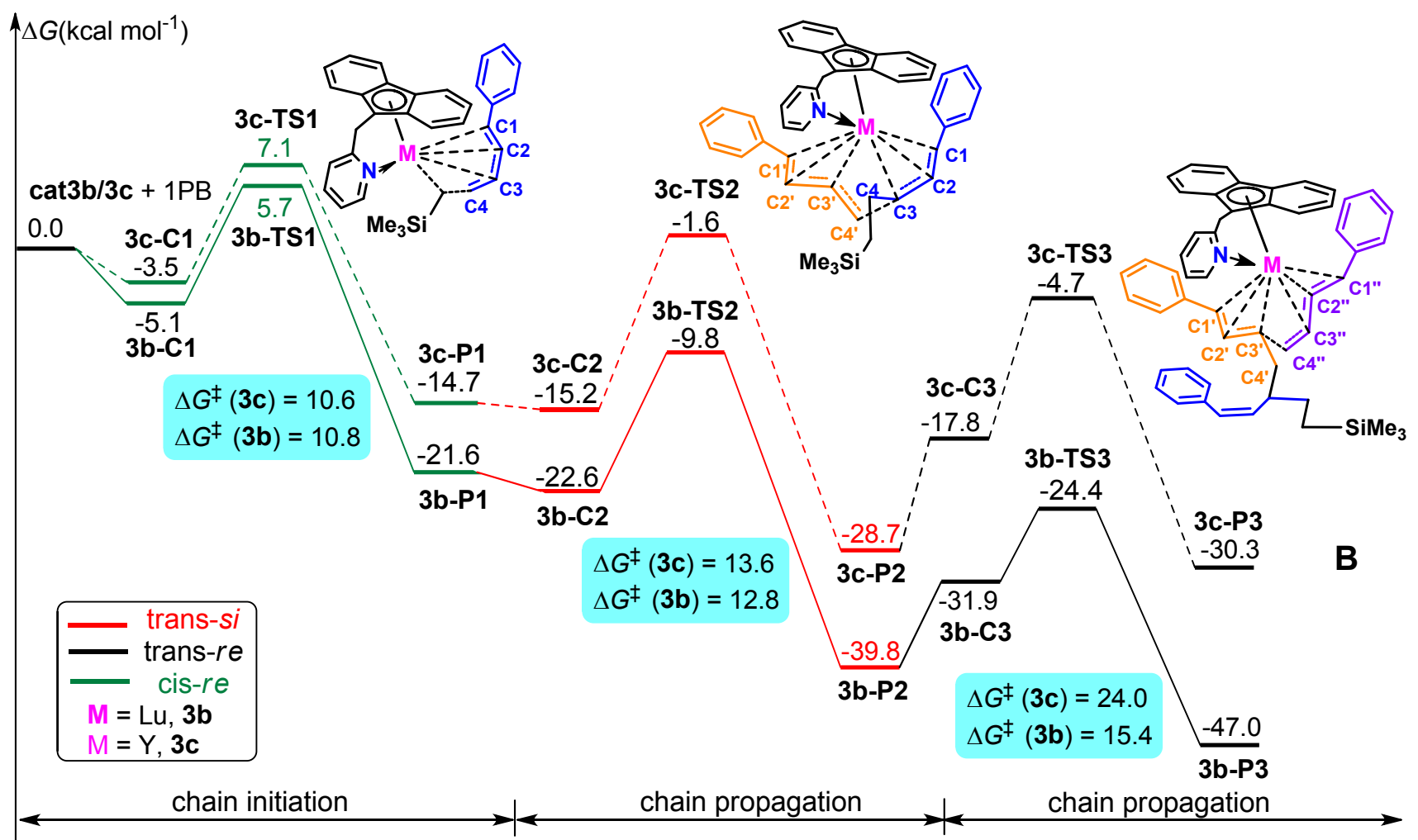

Figure S40 Energy profiles for 1PB polymerization catalyzed by species $\mathbf{3 b}$ and $\mathbf{3 c}$ at chain initiation and propagation stages. Energies are relative to corresponding cationic species cat $3 \mathbf{b} / \mathbf{3 c}$ and $1 \mathrm{~PB}$.

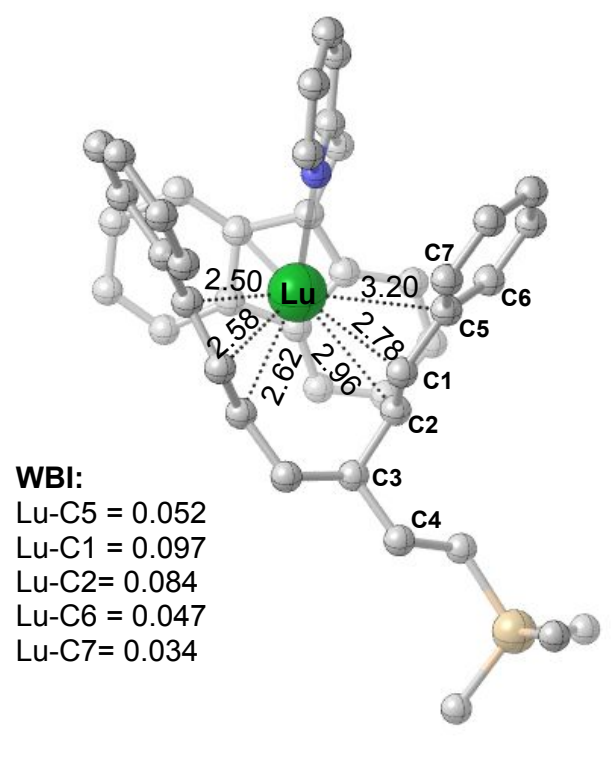

3b-P2 trans-si

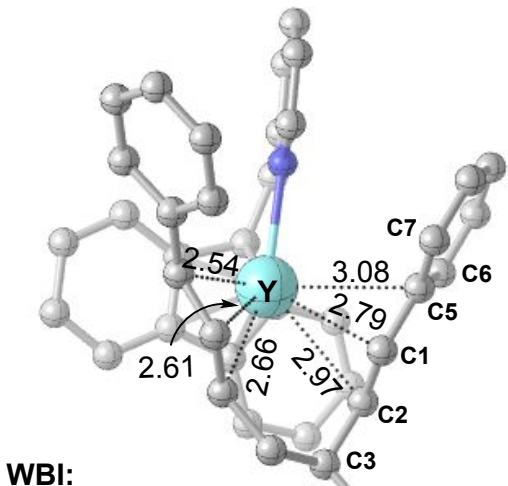

$\mathrm{Y}-\mathrm{C} 5=0.071$

$\mathrm{Y}-\mathrm{C} 1=0.114$

$\mathrm{Y}-\mathrm{C} 2=0.100$

$\mathrm{Y}-\mathrm{C} 6=0.059$

$\mathrm{Y}-\mathrm{C} 7=0.037$

Figure S41 Optimized structures of $\mathbf{3 b} / \mathbf{3 c}-\mathbf{P} \mathbf{2}_{\text {trans-si }}$ in Figure 1 and S39. All hydrogen atoms are omitted for clarity. 


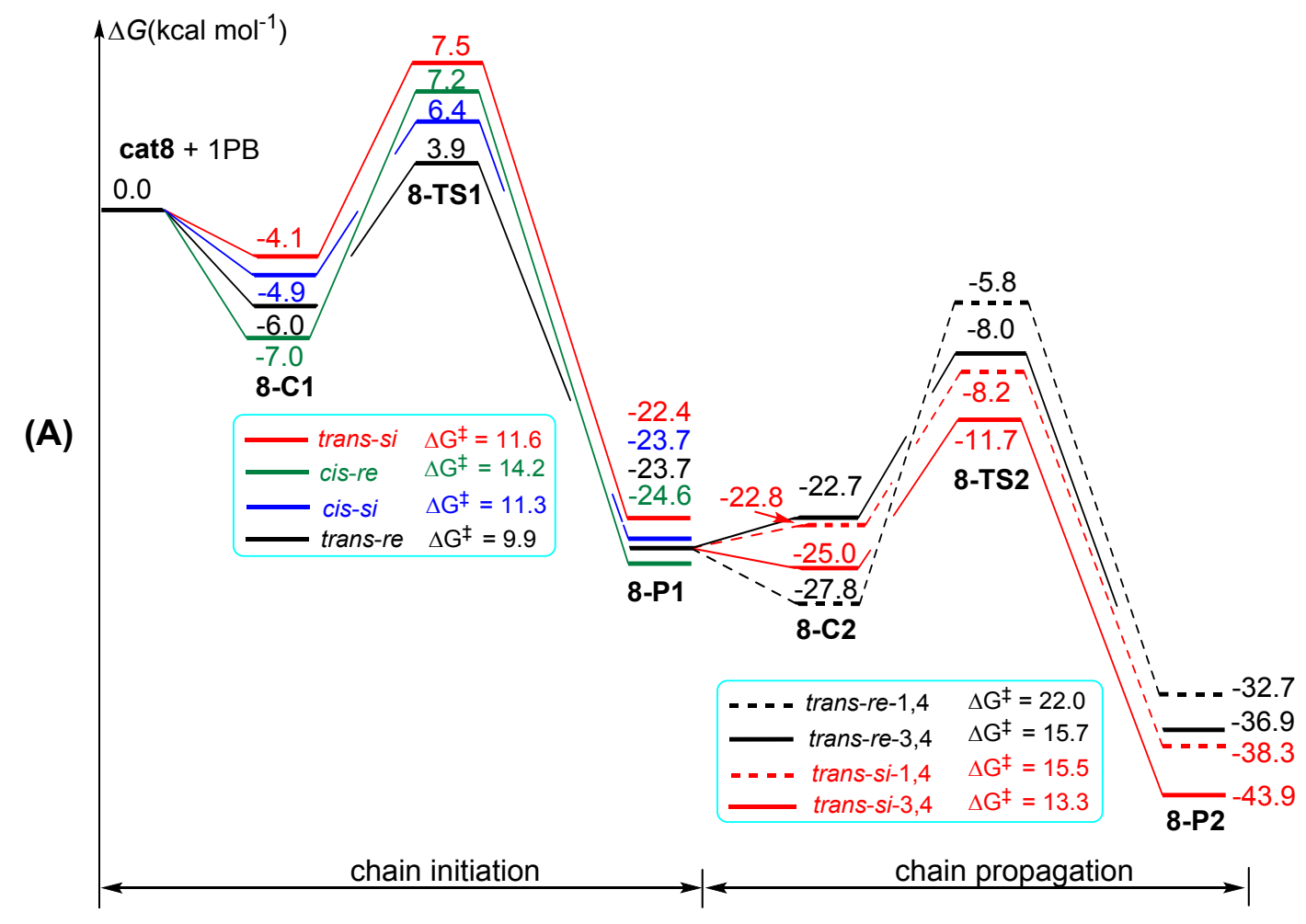

(B)

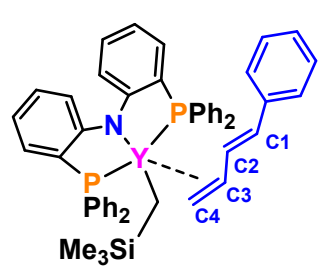

8-C1 $1_{\text {trans-re }}$

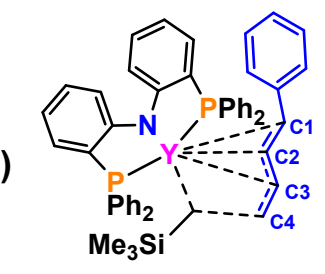

8-TS1 $1_{\text {trans-re }}$

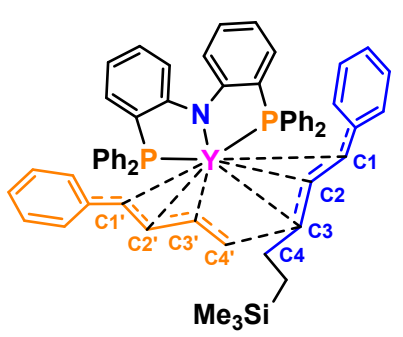

8-TS2 ${ }_{\text {trans-si-34 }}$

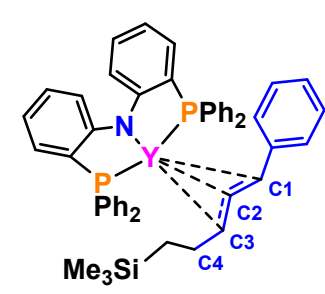

8-P1 trans-re

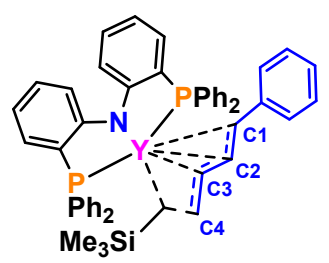

8-TS1 $1_{\text {trans-si }}$

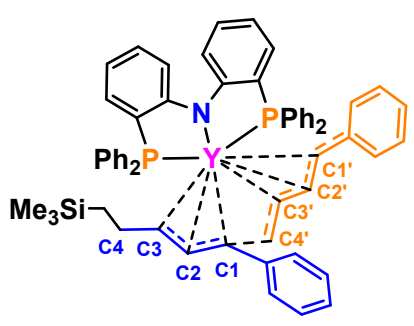

8-TS2 ${ }_{\text {trans-si-14 }}$
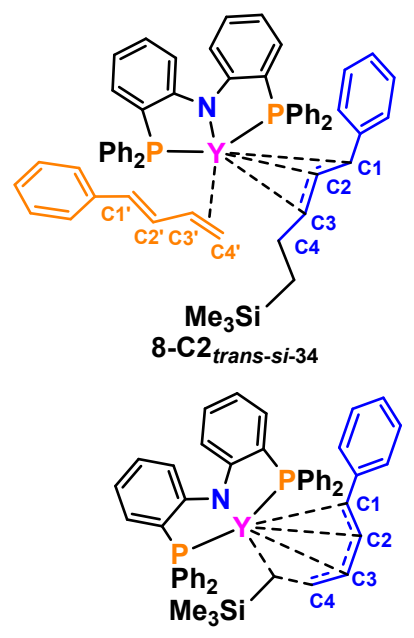

8-TS1 $1_{\text {cis-re }}$

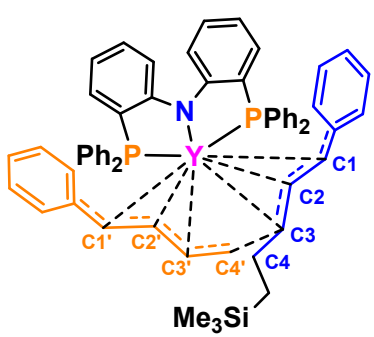

8-TS2 $2_{\text {trans-re-34 }}$
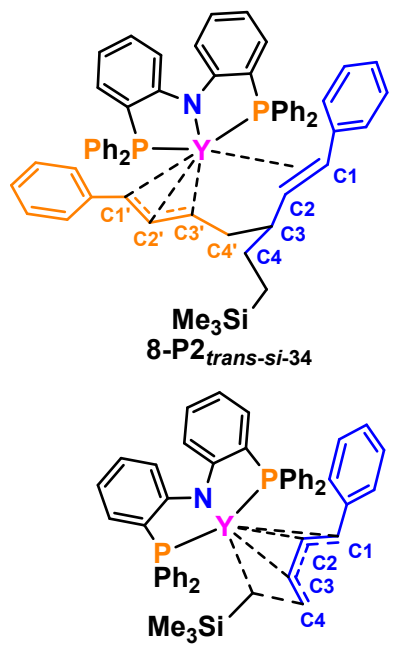

8-TS1 $1_{\text {cis-si }}$

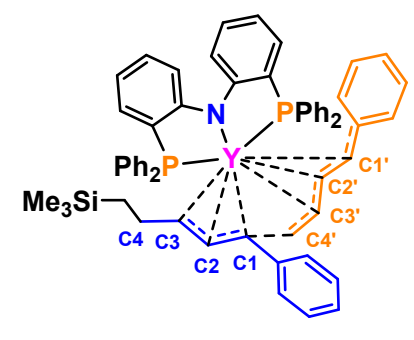

8-TS2 trans-re-14 $_{\text {}}$ 


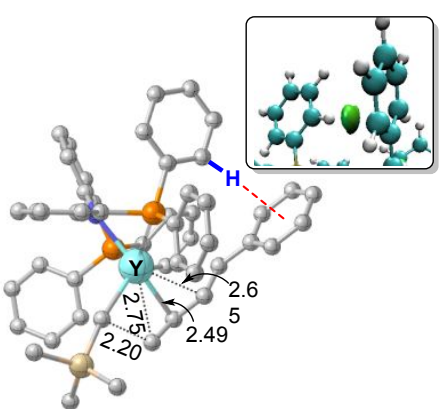

8-TS $1_{\text {trans-re }}$

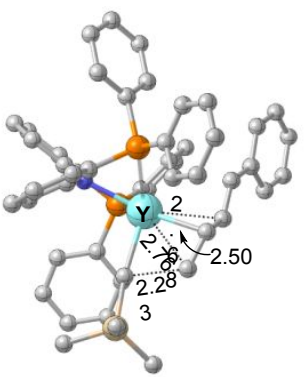

8-TS $1_{\text {trans-si }}$

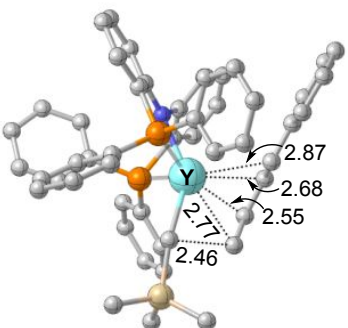

8-TS $1_{\text {cis-re }}$

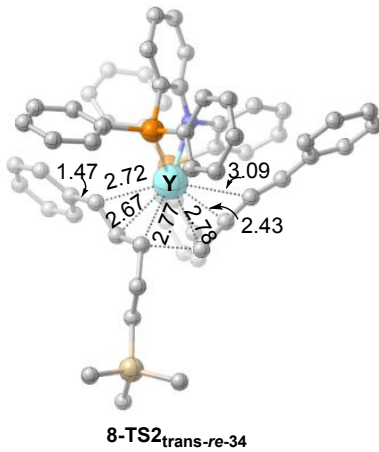

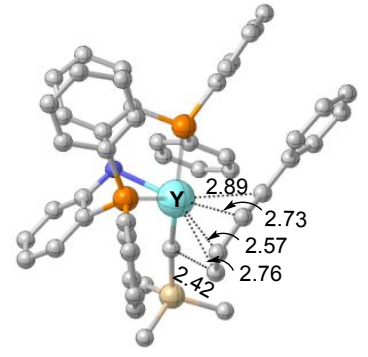

8-TS1 $1_{\text {cis-si }}$

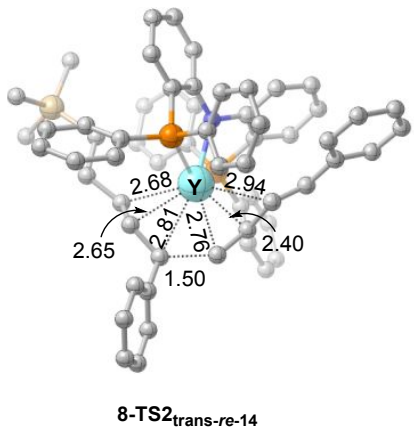

Figure S42. Energy profiles (A), key structures (B) and Optimized structures of transition states (C) of the 1PB polymerization at chain initiation and propagation stages catalyzed by species $\mathbf{8}$. Energies are relative to corresponding cationic species cat8 and 1PB.
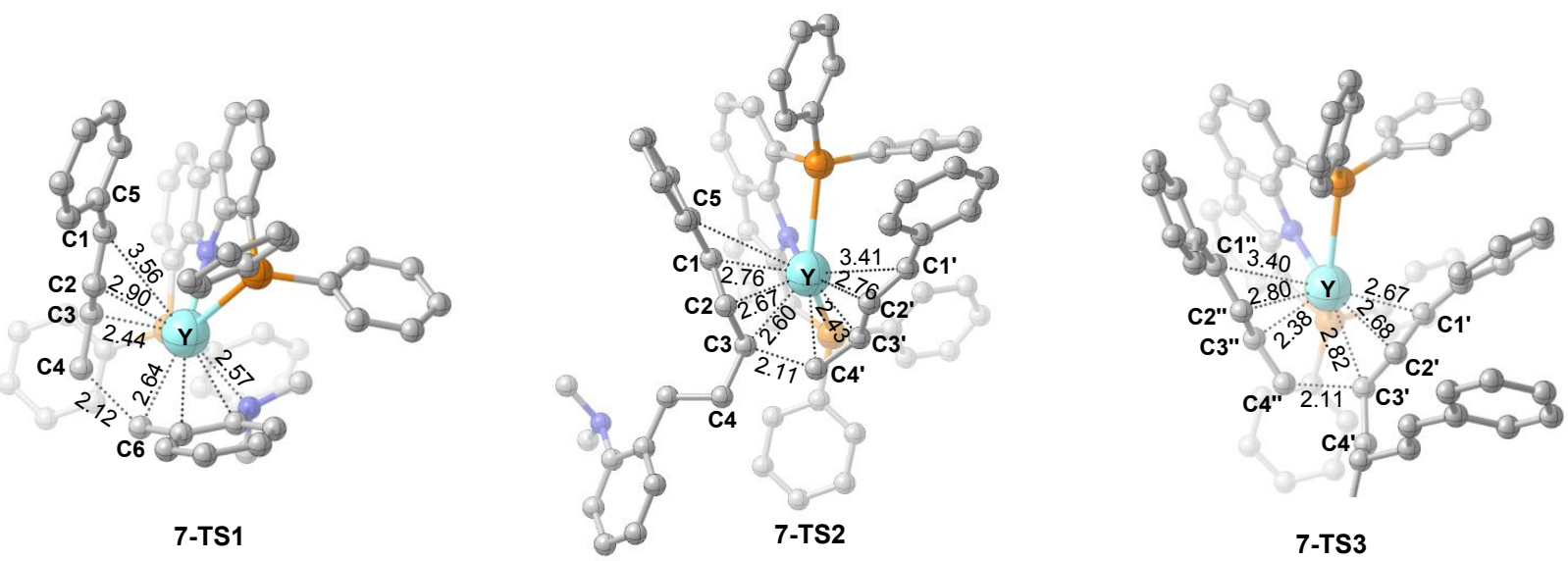

Figure S43. Optimized structures of transition states involved in Figure 2. All hydrogen atoms are omitted for clarity. 